\title{
Non-steady-state, non-uniform transpiration rate and leaf anatomy effects on the progressive stable isotope enrichment of leaf water along monocot leaves
}

\author{
J. OGÉE ${ }^{1}$, M. CUNTZ ${ }^{2,3}$, P. PEYLIN ${ }^{4} \&$ T. BARIAC ${ }^{4}$ \\ ${ }^{1}$ Ephyse (Functional Ecology and Environmental Physics), INRA, Bordeaux, BP 8133883 Villenave d'Ornon cedex, France, \\ ${ }^{2}$ Environmental Biology Group, Research School of Biological Sciences, Australian National University, Canberra, Australia, \\ ${ }^{3}$ Max-Planck Institute for Biogeochemistry, Jena, Germany, and ${ }^{4}$ BioMCO, CNRS/INRA/UPMC, Grignon, France
}

\begin{abstract}
This study focuses on the spatial patterns of transpirationdriven water isotope enrichment $\left(\Delta_{\mathrm{lw}}\right)$ along monocot leaves. It has been suggested that these spatial patterns are the result of competing effects of advection and (back-)diffusion of water isotopes along leaf veins and in the mesophyll, but also reflect leaf geometry (e.g. leaf length, interveinal distance) and non-uniform gas-exchange parameters. We therefore developed a two-dimensional model of isotopic leaf water enrichment that incorporates new features, compared with previous models, such as radial diffusion in the xylem, longitudinal diffusion in the mesophyll, non-uniform gas-exchange parameters and non-steady-state effects. The model reproduces well all published measurements of $\Delta_{\mathrm{lw}}$ along monocot leaf blades, except at the leaf tip and given the uncertainties on measurements and model parameters. We show that the longitudinal diffusion in the mesophyll cannot explain the observed reduction in the isotope gradient at the leaf tip. Our results also suggest that the observed differences in $\Delta_{\mathrm{lw}}$ between $\mathrm{C}_{3}$ and $\mathrm{C}_{4}$ plants reflect more differences in mesophyll tortuosity rather than in leaf length or interveinal distance. Mesophyll tortuosity is by far the most sensitive parameter and different values are required for different experiments on the same plant species. Finally, using new measurements of non-steady-state, spatially varying leaf water enrichment we show that spatial patterns are in steady state around midday only, just as observed for bulk leaf water enrichment, but can be easily upscaled to the whole leaf level, regardless of their degree of heterogeneity along the leaf.
\end{abstract}

Key-words: carbon cycle; deuterium; evaporation; oxygen 18 ; water cycle.

\section{INTRODUCTION}

Several studies have shown that there can be strong spatial variations in the stable isotope composition $\left({ }^{18} \mathrm{O} /{ }^{16} \mathrm{O}\right.$ and

Correspondence: Jérôme Ogée. Fax: +33 (0)5 571224 20; e-mail: ogee@pierroton.inra.fr
$\mathrm{D} / \mathrm{H}$ ) of water along a single leaf, with a general isotopic enrichment from base to tip and from the centre to the edges of the leaf (Bariac 1987; Yakir, DeNiro \& Rundel 1989; Luo \& Sternberg 1992; Bariac et al. 1994a; Wang \& Yakir 1995; Helliker \& Ehleringer 2000; Gan et al. 2002, 2003; Affek, Krisch \& Yakir 2006). This progressive isotopic enrichment is particularly large in monocot leaves (grasses). It can be as high as $+50 \%$ o, especially in $\mathrm{C}_{4}$ plants (Helliker \& Ehleringer 2000). Even in the veins, water gets enriched as one moves along the leaf blade by as much as $+10 \%$ o (Wang \& Yakir 1995; Gan et al. 2002, 2003). Understanding the mechanisms that govern these spatial patterns is needed to scale the results from the leaf to the canopy and beyond. Several studies have also shown that these spatial patterns in leaf water isotope enrichment are partly reflected in the isotope composition of leaf cellulose (Helliker \& Ehleringer 2002; Gan et al. 2003; Affek et al. 2006; Wright \& Leavitt 2006), which therefore records variations in the climate or water availability during leaf development. This leaf cellulose signal could therefore be used to better understand leaf growth and might be potentially valuable also in land-use change impact studies, genetic crop amelioration programmes or for regional climate reconstructions. Yet there is still a need to determine how variability in the isotopic composition of leaf water affects the isotopic composition imprinted in leaf cellulose (Helliker \& Ehleringer 2002).

At the origin of leaf water isotopic enrichment are two isotope fractionation effects that occur during leaf transpiration: (1) the phase change from liquid water to water vapour and (2) the gaseous diffusion between the intercellular air space in the leaf mesophyll and the outer atmosphere (e.g. Dongmann et al. 1974; Farquhar et al. 1989; Bariac, Jusserand \& Mariotti 1990; Flanagan, Comstock \& Ehleringer 1991; Farquhar \& Lloyd 1993; Flanagan 1993). Assuming that the air in the intercellular space is saturated with water vapour and in thermal equilibrium with the liquid phase, the isotope ratio of water vapour in the air space is equal to the isotope ratio of liquid water at the evaporative sites $\left(R_{\mathrm{e}}\right)$ divided by the liquid-vapour equilibrium isotopic fractionation $\alpha^{+}=1+\varepsilon^{+}(>1)$. The latter gives the extent to which the water in the liquid phase is enriched 
in isotopes compared to the water in the vapour phase and depends on temperature. For oxygen-18 and over the range $10-40{ }^{\circ} \mathrm{C}$, it has a mean around $9.4 \pm 0.8 \%$ and a slope of about $-0.08 \pm 0.01 \% \mathrm{~K}^{-1}$ (Majoube 1971 ; Horita \& Wesolowski 1994). Water vapour diffuses out of the leaf mainly through the stomatal pores due to strong water vapour gradients between the air space inside the leaf mesophyll and the outer air. Leaf transpiration $E$ $\left(\mathrm{mol} \mathrm{m} \mathrm{m}^{-2} \mathrm{~s}^{-1}\right)$ is then classically expressed as

$$
E=g_{\mathrm{t}} \cdot\left(w_{\mathrm{i}}-w_{\mathrm{a}}\right),
$$

where $w_{\mathrm{i}}\left(\mathrm{mol} \mathrm{mol}^{-1}\right)$ and $w_{\mathrm{a}}\left(\mathrm{mol} \mathrm{mol}^{-1}\right)$ are the vapour pressures of the air at the sites of evaporation and in ambient air, respectively, and $g_{\mathrm{t}}\left(\mathrm{mol} \mathrm{m}^{-2} \mathrm{~s}^{-1}\right)$ is the total conductance to water vapour diffusion through the stomata and the leaf boundary layer. The same flux-gradient scheme is used for the heavier isotope:

$$
R_{\mathrm{E}} E=\frac{g_{\mathrm{t}}}{\alpha_{\mathrm{k}}} \cdot\left(\frac{R_{\mathrm{e}}}{\alpha^{+}} w_{\mathrm{i}}-R_{\mathrm{v}} w_{\mathrm{a}}\right)
$$

where $R_{\mathrm{E}}$ is the isotope signature of the transpiration flux, $R_{\mathrm{v}}$ is the isotope ratio of water vapour in ambient air and $\alpha_{\mathrm{k}}=1+\varepsilon_{\mathrm{k}}(>1)$ is the overall kinetic fractionation associated with water vapour diffusion in air. The latter can be related to the kinetic fractionation for purely molecular diffusion $\varepsilon_{\mathrm{k}, 0}$ and the boundary-layer $\left(g_{\mathrm{b}}, \mathrm{mol} \mathrm{m}^{-2} \mathrm{~s}^{-1}\right)$ and stomatal $\left(g_{\mathrm{s}}, \mathrm{mol} \mathrm{m}^{-2} \mathrm{~s}^{-1}\right)$ conductances (Farquhar et al. 1989; Flanagan et al. 1991):

$$
\varepsilon_{\mathrm{k}}=\frac{\varepsilon_{\mathrm{k}, 0} g_{\mathrm{b}}+\varepsilon_{\mathrm{k}, 0}^{2 / 3} g_{\mathrm{s}}}{g_{\mathrm{b}}+g_{\mathrm{s}}} .
$$

Values for $\varepsilon_{\mathrm{k}, 0}$ are expected to be around $28.5-32 \%$ for $\mathrm{H}_{2}{ }^{18} \mathrm{O}$ and $16-25 \%$ o for HDO, and are independent of temperature (Merlivat 1978; Cappa et al. 2003). Inserting Eqn 1 into Eqn 2 leads to

$R_{\mathrm{E}}=\frac{R_{\mathrm{e}}-h \alpha^{+} R_{\mathrm{v}}}{\alpha_{\mathrm{k}} \alpha^{+}(1-h)}$

with $h=w_{\mathrm{a}} / w_{\mathrm{i}}$. This can be considered as the air relative humidity calculated at leaf temperature.

Equation 4 is generally assumed to be valid at any time and any point on the leaf surface. At steady state, and if we neglect the export of water in phloem (Farquhar \& Cernusak 2005), the flux-weighted mean isotope signature of the transpiration rate over the leaf surface is equal to the isotope ratio of source water entering the leaf $\left(R_{\mathrm{S}}\right)$, that is, $\bar{R}_{\mathrm{E}}=R_{\mathrm{S}}$ where the over bar denotes the flux-weighted mean over the leaf surface. The mean isotope ratio of leaf water at the sites of evaporation is then given by the so-called CraigGordon equation (Dongmann et al. 1974; Farquhar et al. 1989):

$\bar{R}_{\mathrm{e}}=\alpha^{+}\left[\alpha_{\mathrm{k}}(1-h) R_{\mathrm{s}}+h R_{\mathrm{v}}\right] \equiv R_{\mathrm{C}}$ which is Eqn 4 averaged over the leaf surface and in which we replaced $\bar{R}_{\mathrm{E}}$ by $R_{\mathrm{s}}$ and assumed that all other parameters, notably $\alpha^{+}$and $\alpha_{\mathrm{k}}$, are uniform.

Locally, $R_{\mathrm{E}}$ may be greater than $R_{\mathrm{s}}$ even in the steady state because water in the veins becomes enriched as we move away from the leaf base. At any point on the leaf surface, the maximum possible value for $R_{\mathrm{E}}$ is the local maximum in the liquid phase $R_{\mathrm{e}}$. The maximum leaf water enrichment $R_{\mathrm{M}}$ can then be obtained by setting $R_{\mathrm{E}}=R_{\mathrm{e}}=R_{\mathrm{M}}$ in Eqn 4 (Farquhar \& Gan 2003):

$$
R_{\mathrm{M}}=\frac{h \alpha^{+} R_{\mathrm{v}}}{1-\alpha_{\mathrm{k}} \alpha^{+}(1-h)} .
$$

The isotope ratio of leaf water across the leaf is then expected to lie between the isotope ratio of source water $R_{\mathrm{s}}$ and this maximum value $R_{\mathrm{M}}$. (Note that, if the fractionation factors $\varepsilon_{\mathrm{k}}$ and $\varepsilon^{+}$are non-uniform along the leaf, then $R_{\mathrm{M}}$ should be defined as the upper bound of all possible values taken by Eqn 6).

The observed isotopic enrichment in the leaf veins is due to the intrusion of enriched water from the mesophyll (Yakir 1992; Wang \& Yakir 1995; Helliker \& Ehleringer 2000; Gan et al. 2002). Indeed, despite the large advective transpirational flux towards the stoma, back-diffusion of isotopically enriched leaf water from the evaporative sites occurs towards the vein (Farquhar \& Lloyd 1993) and ultimately enriches the vein water. This enriched vein water travels up the leaf providing source water to the next stomatal complex, and the vein water is further enriched. Yakir (1992) and Wang \& Yakir (1995) therefore compared the flow of water in leaves to a string of interconnected and evaporating lakes and proposed to use the so-called 'string-of-lakes' model (Gat \& Bowser 1991) in order to explain the progressive enrichment of leaf water from base to tip. This model has been further tested by Helliker \& Ehleringer (2000) on a variety of $\mathrm{C}_{3}$ and $\mathrm{C}_{4}$ grasses. However, to fit their measurements, the authors had to assume that leaf transpiration was not uniform along the leaf blade, with greater values at the base (Helliker \& Ehleringer 2000). They also assumed that the leaf could be divided into a discrete, finite number of evaporating elements, corresponding to the number of segments to which the leaf was cut before isotopic analysis. Gan et al. (2002) noted that this value was rather arbitrary and could greatly influence the isotopic enrichment along the leaf. Furthermore, they suggested a continuous description of the evaporative elements seemed more appropriate for leaves, and this leads to the 'desert river' model (Fontes \& Gonfiantini 1967; Gan et al. 2002). In this model, the isotope ratio $R_{\mathrm{lw}}(l)$ of leaf water at a distance $l$ from the leaf base depends mostly on relative humidity $h$ (Farquhar \& Gan 2003):

$$
\frac{R_{\mathrm{M}}-R_{\mathrm{lw}}(l)}{R_{\mathrm{M}}-R_{\mathrm{s}}}=\left(1-\frac{l}{l_{\max }}\right)^{\frac{h^{\prime}}{1-h^{\prime}}},
$$


where $h^{\prime}=1-\alpha_{\mathrm{k}} \alpha^{+}(1-h)$ and $l_{\max }$ is the total leaf length. (Note that Eqn 7 assumes uniform values of the fractionation factors).

The desert-river model does not distinguish between the isotope composition of water in the leaf veins and in the mesophyll. Moreover, by integrating Eqn 7 over the leaf length, we can show that the mean leaf water isotope ratio predicted by this model is simply the Craig-Gordon value $R_{\mathrm{C}}$ (Eqn 5). Yet, several studies have shown that $R_{\mathrm{C}}$ overestimates the isotope ratio of bulk leaf water, even after removal of all lateral veins (e.g. Bariac et al. 1990; Flanagan et al. 1991; Flanagan 1993; Roden \& Ehleringer 1999). This is because Eqn 5 is valid at the sites of evaporation only and not across the entire leaf thickness and the leaf mesophyll is not well homogenized. For this reason, Farquhar \& Lloyd (1993) suggested that back-diffusion of enriched water, from the sites of evaporation to other parts of the mesophyll and into the veins, is too slow to ensure a complete mixing and leads to a non-zero isotopic gradient in the mesophyll. The magnitude of the gradient depends on the relative importance of convection (proportional to $E$ ) and (back-)diffusion and is characterized by a 'radial' Péclet number $\wp_{\mathrm{m}}$ (Farquhar \& Lloyd 1993):

$\wp_{\mathrm{m}}=\frac{E L_{\mathrm{m}}}{C D}$,

where $C\left(=55.5 \times 10^{3} \mathrm{~mol} \mathrm{~m}^{-3}\right)$ is the molar concentration of liquid water, $D\left(\mathrm{~m}^{2} \mathrm{~s}^{-1}\right)$ is the tracer diffusivity of $\mathrm{H}_{2}{ }^{18} \mathrm{O}$ (or HDO) in liquid water and $L_{\mathrm{m}}$ is the effective mixing length in the mesophyll. [Note that, compared to Farquhar \& Lloyd (1993), we added a subscript 'm' in $\wp_{\mathrm{m}}$ and $L_{\mathrm{m}}$ to recall that they are relative to the mesophyll reservoir]. The effective mixing length $L_{\mathrm{m}}$ is expected to be many times greater than the actual mesophyll thickness $r_{\mathrm{m}}$ (of the order of $100 \mu \mathrm{m}$ ) because water is not moving linearly inside the mesophyll but rather follows a tortuous path (Farquhar \& Lloyd 1993). Barbour \& Farquhar (2003) used a realistic anatomical representation of a wheat leaf mesophyll in order to estimate the tortuosity of the water path in this reservoir. They estimated the effective mixing length to be $10^{2}$ to $10^{3}$ greater than $r_{\mathrm{m}}$, depending on the water path and the location of the evaporation sites. Yet, only one single bulk value is usually used for $L_{\mathrm{m}}$ and it is expected to be constant for a given leaf (e.g. Barbour et al. 2000; Farquhar \& Gan 2003).

In order to account for this incomplete mixing in the mesophyll and distinguish between the isotopic composition in the leaf veins and in the mesophyll, Gan et al. (2002) and Farquhar \& Gan (2003) modified Eqn 7. They also extended the model to account for possible variation in transpiration rates along the leaf (but uniform values of the fractionation factors). The isotope ratios of leaf water in the leaf 'longitudinal xylem' $R_{\mathrm{x}}(l)$ then become (Farquhar \& Gan 2003)

$$
\frac{R_{\mathrm{M}}-R_{\mathrm{x}}(l)}{R_{\mathrm{M}}-R_{\mathrm{s}}}=\left(\frac{I(l)}{I_{\max }}\right)^{\mathrm{k}},
$$

with

$$
\begin{aligned}
I(l) & =\int_{l}^{l_{\max }} E\left(l^{\prime}\right) \cdot d l^{\prime}, \quad I_{\max }=\int_{0}^{l_{\max }} E\left(l^{\prime}\right) \cdot d l^{\prime} \text { and } \\
k & =\frac{h^{\prime}}{e^{\wp_{\mathrm{m}}}-h^{\prime}} .
\end{aligned}
$$

In addition, the local isotope ratio in the leaf mesophyll $R_{\mathrm{m}}(l)$ and the local isotope signature of the evaporation flux $R_{\mathrm{E}}(l)$ are given by (Farquhar \& Gan 2003):

$$
\frac{R_{\mathrm{m}}(l)-R_{\mathrm{E}}(l)}{R_{\mathrm{x}}(l)-R_{\mathrm{E}}(l)}=\frac{1-e^{-\wp_{\mathrm{m}}}}{\wp_{\mathrm{m}}}
$$

and

$$
R_{\mathrm{E}}(l)=\frac{e^{\wp_{\mathrm{m}}} R_{\mathrm{x}}(l)-\alpha^{+} h R_{\mathrm{v}}}{e^{\wp_{\mathrm{m}}}-h^{\prime}}
$$

where $\wp_{\mathrm{m}}$ is now expressed in terms of local leaf evaporation $E(l)$. [Note that, in contrast to Farquhar \& Gan (2003), we do not consider the 'veinlets' coming out of the leaf veins into the mesophyll as a separate reservoir. Therefore, $\wp_{\mathrm{m}}$ is the only radial Péclet number to consider in the mesophyll]. Gan et al. (2003) further tested this model and concluded that Eqn 9a was able to describe the measured progressive enrichment of leaf water in the midrib and the marginal lateral vein of a maize leaf for up to about $80 \%$ of the leaf length, but the model tended to predict a stronger enrichment at the leaf tip. A similar conclusion had been made by Helliker \& Ehleringer (2000) about the discrete string-of-lakes model tested against bulk leaf water, but this was resolved by allowing leaf transpiration to vary along the leaf. Unfortunately the transpiration rate and its variations were not measured in their experiment so that the shape they used for $E(l)$ could not be validated.

Gan et al. (2003) did suspect possible heterogeneity in leaf transpiration, temperature or light levels but also noted that the back-diffusion of heavy water in the leaf veins (from tip to base) was not accounted for in Eqn 9a and should lead to a reduced enrichment at the leaf tip (Farquhar \& Gan 2003; Gan et al. 2003). Therefore, they modified Eqn 9a in order to incorporate the effect of backdiffusion in the leaf longitudinal xylem. For that, a particular shape for the xylem reservoir had to be specified. The latter was materialized by identical and cylindrical veins (length $l_{\max }$, cross-sectional area $a_{\mathrm{x}}$ ), parallel to the leaf main axis and separated by a fixed distance (denoted by $s_{\mathrm{x}}$ and taken as a measure of vein density; see Fig. 1). As pointed out by Farquhar \& Gan (2003), this representation of the leaf xylem is an approximation of what happens in monocotyledonous (grass) leaves and is much harder to apply to the reticulate network of veins in dicotyledonous leaves. Water movement within each vein was described with a one-dimensional (1D) advection-diffusion equation in the longitudinal direction (coupled to a $1 \mathrm{D}$ advection-diffusion in the radial direction within the mesophyll). The simplified geometry of the leaf xylem allows definition of a longitudinal Péclet number in this reservoir: 


\section{Dicot Monocot}

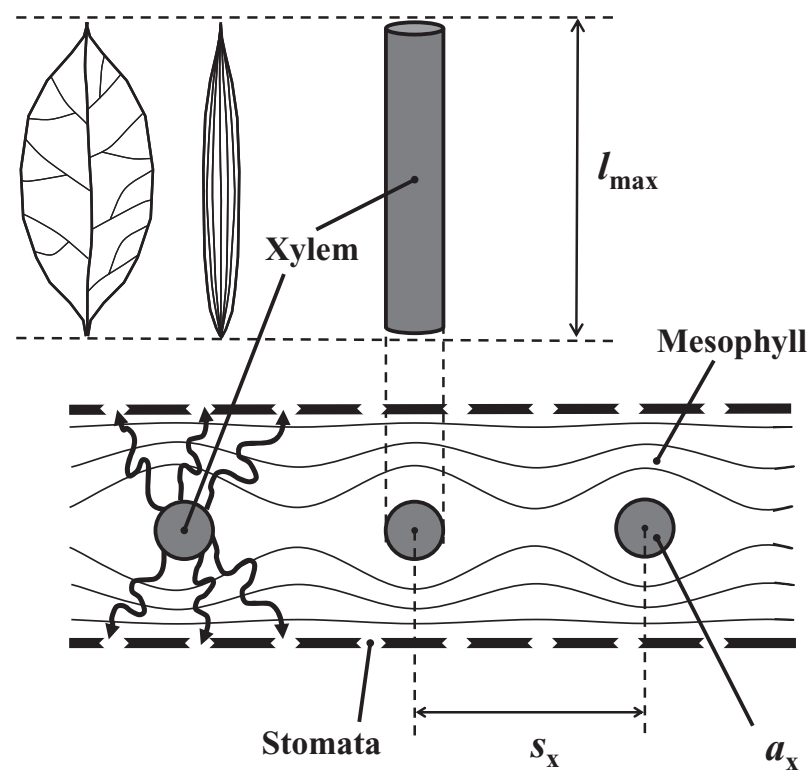

Figure 1. Simplified leaf geometry used by Farquhar \& Gan (2003) to model the progressive enrichment of leaf water in monocotyledonous leaves. The leaf xylem is represented by identical and parallel veins (length $l_{\max }$, cross-sectional area $a_{\mathrm{x}}$ ), oriented from leaf base to leaf tip and separated by a fixed distance $s_{\mathrm{x}}$.

$\wp_{1}=\frac{v_{\mathrm{x} 0} l_{\max }}{D}=\frac{\bar{E} s_{\mathrm{x}} l_{\max }^{2}}{a_{\mathrm{x}} C D}$,

where $v_{\mathrm{x} 0}\left(\mathrm{~m} \mathrm{~s}^{-1}\right)$ is the water velocity at the base of the leaf longitudinal xylem, used here as the velocity scale, and is related to the evaporation rate by means of the steady-state water budget inside the veins: $a_{\mathrm{x}} C v_{\mathrm{x} 0}=\bar{E} S_{\mathrm{x}} l_{\text {max }}$. This longitudinal Péclet number can be easily estimated from anatomical dimensions of the leaf veins and is expected to be of the order of $10^{6}-10^{7}$, regardless of the types of veins (i.e. lateral or intermediate) involved (Gan et al. 2003). This high value for $\wp_{1}$ indicates that longitudinal diffusion should be negligible inside the veins unless the longitudinal advection flux rapidly decreases along the leaf (it is zero at the leaf tip). Indeed, in order to make this new leaf water enrichment model fit to their measurements, Gan et al. (2003) had to use an effective longitudinal Péclet number $\wp_{1}$ much lower $\left(10^{4}\right.$ at low humidity levels and down to $10^{2}$ at high humidity levels) than the estimated value based on anatomical dimensions. They suggested that tapering of the vein diameter $\left(\propto \sqrt{a_{\mathrm{x}}}\right)$ or the transpiration rate $(E)$ towards the leaf tip might partly explain these unexpectedly low Péclet numbers. Barnes, Farquhar \& Gan (2004) further tested these two hypotheses but found that very small $\wp_{1}$ values were still necessary to reproduce the observed water enrichment patterns along the leaf, regardless of the humidity levels. The conclusion of their studies might be biased by the fact that, due to the motivation of getting an analytical solution, Barnes et al. (2004) had to assume an a priori shape of vein diameter variations (linear decrease) or nonuniform transpiration rate (power-law) along the leaf length. For example, a closer analysis of the data used by Gan et al. (2003) to test the validity of Eqn 9a (which assumes $\wp_{1} \rightarrow \infty$ ) shows that strong spatial variations in the transpiration rate alone can explain the reduced enrichment at the leaf tip. This is shown in Fig. 2, where we can also appreciate the need to account for a non-zero radial Péclet number in the mesophyll (top panel). However, different patterns in the transpiration field were used for the midrib and the marginal lateral vein (bottom panel) with variations by a factor 10 between the minimum and the maximum, which is unrealistic because the maize leaf was in a controlled environment. This supports the idea that other processes, not accounted for by current models, are causing a stronger mixing in the longitudinal direction of the leaf, especially at the leaf tip.

Bearing this in mind, Gan et al. (2003) and Barnes et al. (2004) argued that these low $\wp_{1}$ values were justified by the fact that longitudinal water movement in the mesophyll is not accounted for in their model but is likely to occur, and with a much smaller ratio of advection to diffusion, especially at the leaf tip where the isotopic gradient is highest. Furthermore, if water movements in this leaf reservoir occur mainly in symplastic water through water channels or aquaporins, water molecules are then expected to move in single file (Schäffner 1998) and the propagation of isotopic

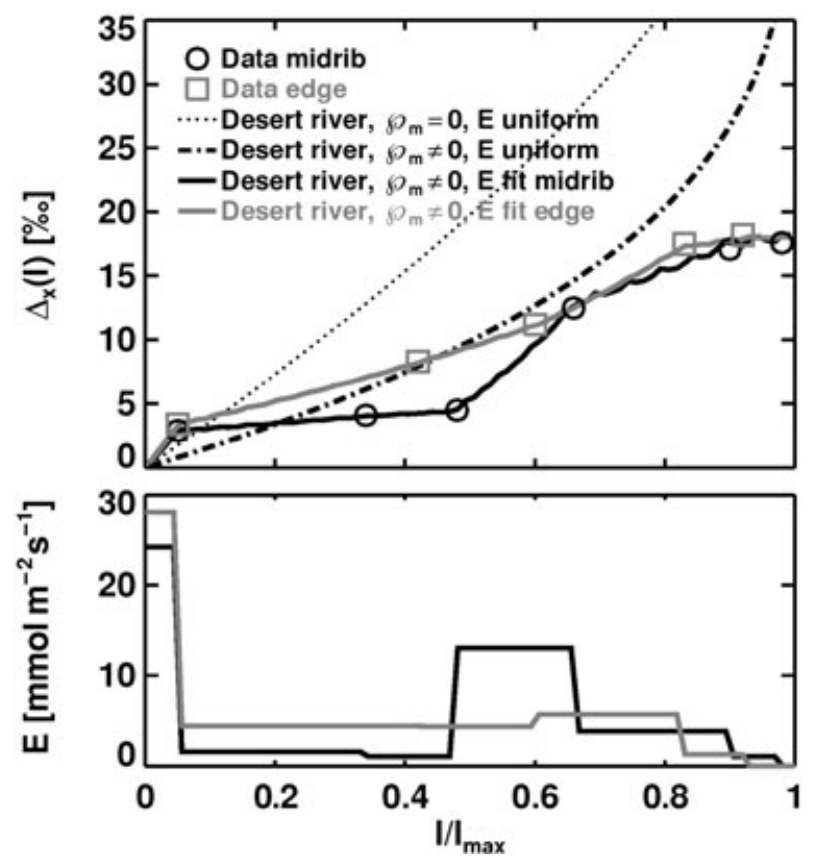

Figure 2. Progressive enrichment $\Delta_{\mathrm{x}}(l)=R_{\mathrm{x}}(l) / R_{\mathrm{s}}-1$ measured in the midrib (circles) or in the marginal lateral vein (squares) of a maize leaf and predicted by the desert-river model with zero (dotted line, Eqn 7 in the text), or non-zero radial Péclet number (Eqn $9 \mathrm{a}$ in the text). Values of $l_{\max }=0.5 \mathrm{~m}, \bar{E}=7 \mathrm{mmol} \mathrm{m}^{-2} \mathrm{~s}^{-1}$, $h=0.399, \varepsilon^{+}=8.4 \%$, $\varepsilon_{\mathrm{k}}=24.4 \%$ o, $\delta_{\mathrm{v}}=-11.6 \%$ VSMOW and $\delta_{\mathrm{s}}=-4.8 \%$ VSMOW were used. Experimental data are redrawn from Gan et al. (2003). 
gradients is still possible but in a fashion similar to Knudsen diffusion, a sort of 'continuous-time random walk' (Reinecke \& Sleep 2002; Zhu \& Schulten 2003). The mixing length $L_{\mathrm{m}}$ appearing in Eqn 8 would therefore not only account for the tortuosity of the water path in the mesophyll but also for the difference between the Knudsen diffusion coefficient and the tracer diffusivity in free water.

Radial movements inside leaf veins exist too and, because the veins are composed of several vessels (Gan et al.2003), the water path during these radial movements is likely to be tortuous as well. Farquhar \& Gan (2003) did not try to model explicitly these radial movements in the leaf veins probably because it raises some difficulties linked to the geometry of the model leaf (Fig. 1), that is, parallel and cylindrical reservoir (the vein) feeding a larger but rather planar reservoir (the mesophyll). However, these radial movements and their possible tortuosity may equally affect the spatial patterns of the isotopic composition of leaf water.

Variations of gas-exchange parameters like leaf temperature or stomatal conductance create a non-uniform transpiration rate $E$ but should also substantially affect $\varepsilon_{\mathrm{k}}$ and $\varepsilon^{+}$ and therefore the spatial patterns of leaf water enrichment. Due to the important turbulent coupling to the atmosphere, leaf temperature gradients should not normally exceed $2-3{ }^{\circ} \mathrm{C}$ in the field, leading to differences in $\varepsilon^{+}$of about $0.3 \%$ between the coolest and the warmest parts of a leaf. However these gradients might be much higher between shaded and sunlit parts of the same leaf. Furthermore, previous studies on spatial variations of stomatal conductance along leaves (Meinzer \& Salendra 1997; Buckley \& Mott 2000; Affek et al. 2006) generally report an increase of this parameter along the leaf by a factor of ca 2. In some extreme experiments, patchy stomatal conductance has been observed in response to strong light variations (e.g. Mott \& Buckley 1998) but, because shaded leaves are never in complete darkness, stomatal heterogeneity should be more limited in the field. Yet, variations by a factor 2 in stomatal conductance already lead to differences in $\varepsilon_{\mathrm{k}}$ of about 1\%o (Eqn 3). If our aim is to explain spatial heterogeneity in the isotopic composition of leaf water, it seems therefore appropriate to take into account the spatial variations of the fractionation factors along the leaf.

Helliker \& Ehleringer (2000) also suggested that leaf length $\left(l_{\max }\right)$ and interveinal distance $\left(s_{\mathrm{x}}\right)$ were key parameters to explain differences of steady-state leaf water enrichment between different species. However, from our current understanding of leaf water enrichment, it is not clear yet how these geometrical parameters influence bulk leaf water enrichment. For example, it is easy to show that Eqns 7 and 9a are both insensitive to these two parameters. A better inspection of the influence of these leaf geometrical parameters on leaf water enrichment is clearly needed.

Finally, it seems important to know how these spatial patterns may vary in time due to changing environmental conditions (Wang \& Yakir 1995; Yakir 1998). Indeed, we know that in the field, the isotopic composition of leaf water is not in steady state, except during a few hours in the early afternoon (Dongmann etal. 1974; Bariac et al. 1991; Harwood et al. 1998; Cernusak, Pate \& Farquhar 2002; Farquhar \& Cernusak 2005). Yet all the models described earlier are valid in the steady state only and are therefore harder to apply in the field. To date, only one study (Yakir 1998) tried to describe how the spatial patterns of isotopic enrichment along leaves could vary in time. However, the geometry of the modelled leaf was very crude and included only one single squared reservoir. A more realistic dynamic and spatially explicit model of leaf water isotopic enrichment is therefore needed.

The objective of this study is to understand the role played in the spatial heterogeneity of the isotopic composition of leaf water by (1) longitudinal diffusion in the mesophyll and radial diffusion in the xylem and their associated tortuosities, (2) non-uniform gas-exchange parameters (leaf temperature and stomatal conductance), (3) leaf geometry $\left(l_{\max }, s_{\mathrm{x}}\right)$ and (4) non-steady-state effects. For that, we extended the model of Farquhar \& Gan (2003) to a fully explicit $2 \mathrm{D} \times 2 \mathrm{D}$ advection-diffusion model that incorporates longitudinal diffusion in the mesophyll but also radial movements in the xylem. We assume that isotopic gradients can be propagated through the leaf mesophyll (in both directions) but we make no assumption concerning the type of diffusion involved. Our model also accounts for possible variations of leaf temperature and stomatal conductance and consequently of the fractionation factors $\alpha_{\mathrm{k}}$ and $\alpha^{+}$. Finally, we extended the model equations to non-steadystate conditions. The model is then solved numerically and tested against previously published data sets of spatially varying leaf water isotopic enrichment measured in the steady state (Helliker \& Ehleringer 2000; Gan et al. 2003; Affek et al.2006), and an unpublished data set measured in the non-steady state. In this paper, we show how different values of the diffusivities or different spatial patterns of leaf temperature or stomatal conductance affect the distribution of leaf water isotopes along the leaf. In particular, we consider if our model can satisfactorily reproduce the observed progressive leaf water isotopic enrichment, even with a high longitudinal Péclet value in the xylem estimated from the anatomical dimensions of the leaf veins. We also examine how leaf geometry influences $\Delta_{\text {lw }}$ and to what extent the model can be used to interpret the progressive leaf water enrichment measured in the non-steady state in the field. A complete description of the model is given in the next section.

\section{MODEL DESCRIPTION}

\section{Leaf geometry and model assumptions}

We adopt the same leaf geometry as in Farquhar \& Gan (2003): the leaf longitudinal xylem is composed of identical and parallel veins with cylindrical shape and oriented from the base to the tip of the leaf (Fig. 1). We denote respectively by $r_{\mathrm{x}}(\mathrm{m})$ and $l_{\max }(\mathrm{m})$ the radial and longitudinal dimensions of the veins (whose cross-sectional area is therefore $a_{\mathrm{x}}=\pi r_{\mathrm{x}}^{2}$ ) and the distance between two adjacent 
veins is $s_{\mathrm{x}}(\mathrm{m})$. We further assume that the volume of the mesophyll, which is crossed by water going out of each vein, has a planar geometry with half-thickness $r_{\mathrm{m}}(\mathrm{m})$. In the following we will assume that $r_{\mathrm{x}}, r_{\mathrm{m}}$ and $s_{\mathrm{x}}$ are constant along the leaf. Including tapering xylem (changing $r_{\mathrm{x}}$ ) is described in Barnes et al. (2004) for steady-state conditions and does not change the principal conclusions but hampers the accessibility. We thus preferred to exclude this effect from our model.

Following Farquhar \& Gan (2003), we assume that the leaf can be decomposed into independent parts defined by one single vein and the mesophyll cells that surround it (Fig. 3). Inside this single vein, we make the simplifying

(a)
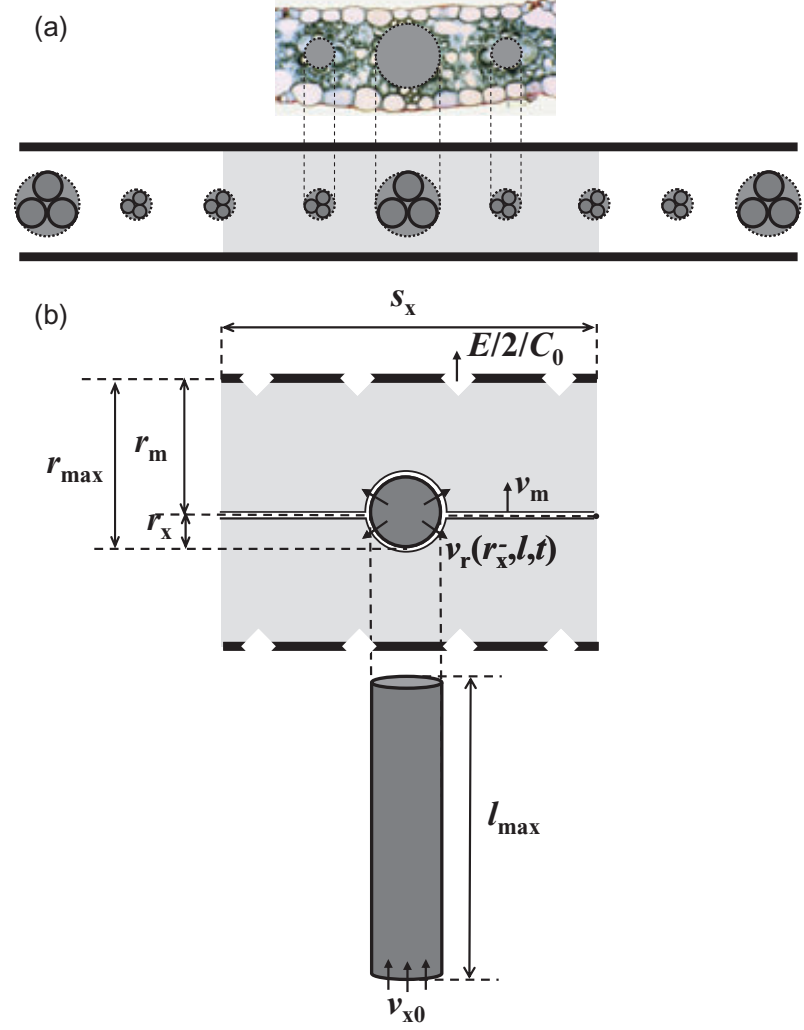

Figure 3. Model leaf geometry. Panel A shows a cross-section of a maize leaf and its schematic representation in terms of longitudinal xylem (dark grey region) and mesophyll (light grey region). Lateral (respectively intermediate) veins are represented by large (respectively small) dotted circles and each individual vessel inside the veins is represented by a solid circle. Panel B shows the cross-section of the leaf as represented in the numerical model. The leaf is divided into independent parts of equal width $s_{\mathrm{x}}$ (equal to the distance between two lateral veins). Each part is made of one single cylindrical vein (with cross-sectional area $a_{\mathrm{x}}$, equal to the total cross-sectional area of all vessels, and an apparent radius $\left.r_{\mathrm{x}}=\sqrt{a_{\mathrm{x}} / \pi}\right)$ and the mesophyll (with thickness $2 r_{\mathrm{m}}$ ). The flux of water going out of the leaf vein at $r_{\mathrm{x}}$ is assumed to be feeding the mesophyll from the middle of the leaf (with the help of intermediate veins, not explicitly represented in our model). The total path length that can be travelled by water molecules is then $r_{\max }=r_{\mathrm{x}}+r_{\mathrm{m}}$. Photo credit: D. Vodopitch

(http://www3.baylor.edu/ Darrell_Vodopich/). assumption that water movements have symmetry of revolution around the longitudinal axis thereby neglecting circular movements around the $l$-axis. Inside the mesophyll, we assume that water movements rapidly adopt a planar symmetry with respect to the leaf surface, and therefore neglect any radial movement that is not normal to the leaf plane. It is a rough approximation of what happens in amphistomatous leaves where stomata are almost equally distributed between the upper and lower mesophyll. Water is then allowed to move in the radial or longitudinal directions in the vein and in a direction normal to the leaf surface or parallel to the vein axis in the mesophyll. In this reservoir, longitudinal advection is neglected so that water is advected in the radial direction only, but diffusion takes place in both directions.

Because $E$ is expressed per leaf area, the total amount of water transpired is $E \times s_{\mathrm{x}} l_{\max }$, but this transpiration occurs equally on both sides of the leaf so that the actual advection flux in the mesophyll is only E/2. Strictly speaking, this equality is true only if we can neglect water storage in the vapour phase inside the mesophyll, which is likely to be the case because the molar concentration in the vapour phase is about $10^{4}-10^{5}$ smaller than $C$. We further assume that the tortuosity of the water path in the mesophyll is the same in all directions. A single tortuosity factor $\kappa_{\mathrm{m}}(<1)$ is then used to compute an effective diffusivity in the mesophyll: $D_{\mathrm{m}}=\kappa_{\mathrm{m}} D\left(\mathrm{~m}^{2} \mathrm{~s}^{-1}\right)$. This expression for $D_{\mathrm{m}}$ is taken from studies on movements of water isotopes in saturated soils (e.g. Mathieu \& Bariac 1996) and is assumed to be valid also in the mesophyll, even though the value of $\kappa_{\mathrm{m}}$ is much smaller in the leaf mesophyll $\left(10^{-2}-10^{-3}\right.$; see Barbour \& Farquhar 2003) than in saturated soils (ca 0.6). The radial Péclet number in the mesophyll can be rewritten in terms of this tortuosity factor and the radial velocity $E / 2 / C$ :

$\wp_{\mathrm{m}}=\frac{E r_{\mathrm{m}}}{2 C D_{\mathrm{m}}}=\frac{E}{C D} \cdot \frac{r_{\mathrm{m}}}{2 \kappa_{\mathrm{m}}}$

and the effective mixing length $L_{\mathrm{m}}$ is then related to the mesophyll half-thickness $r_{\mathrm{m}}$ through

$L_{\mathrm{m}}=\frac{r_{\mathrm{m}}}{2 \kappa_{\mathrm{m}}}$

In the leaf longitudinal xylem, a tortuosity factor $\kappa_{\mathrm{x}}(<1)$ is also used in order to account for a possible tortuous path of water in this reservoir, but for radial diffusion only.

The sites of evaporation are assumed to be located at a fixed distance $r_{\mathrm{e}}(\mathrm{m})$ from the vein main axis, equal to $r_{\max }=r_{\mathrm{m}}+r_{\mathrm{x}}$ (see Fig. 3), assuming that evaporation most likely occurs near the stomatal cavities. When water evaporates faster than the leaf veins can refill the mesophyll cells, the total volume of liquid water in the mesophyll decreases (Yakir 1998; Cernusak et al. 2002; Farquhar \& Cernusak 2005). For simplicity, changes in leaf water volume will be neglected in the following and volumetric leaf water content is taken as one across the mesophyll and the leaf veins. 
All variables in our model are functions of time $t$ and the radial and the spatial coordinates $r$ and $l$, respectively. Our objective is to compute the isotope ratio $R(r, l, t)$ of leaf water at time $t$ and at any distance $r$ from the vein main axis and any distance $l$ from the leaf base. We also integrate all equations along the $l$ - or $r$-axis in order to get equations for bulk leaf water isotope values in the xylem and the mesophyll and to compare them to previous models. Mean values along the $l$-axis will be denoted by an over bar while mean values along the $r$-axis will be denoted by subscript ' $x$ ' in the xylem and subscript ' $m$ ' in the mesophyll.

\section{Water velocity field}

Water movements are characterized by a velocity field $\boldsymbol{v}=\left\{v_{\mathrm{r}}, v_{\mathrm{l}}\right\}\left(\mathrm{m} \mathrm{s}^{-1}\right)$ in the radial and longitudinal directions, respectively. Because water concentration in the leaf is uniform and constant in time (no water storage), the local continuity equation for leaf water reduces to

$\nabla \cdot \boldsymbol{v}=0$,

that is, the divergence of the velocity field is zero. Equation 13 is just the conservation of mass for an incompressible flow. To compute the velocity field, the Navier-Stokes equations (which represent the conservation of momentum) should be used together with Eqn 13. We would then have a system of three equations and three unknowns $\left(v_{\mathrm{r}}, v_{1}\right.$ and the water pressure field) with a unique solution. However, with a few simplifications, the mean velocity field can be derived more simply, as described next.

As explained, we first neglect longitudinal advection in the mesophyll: $v_{1}(r, l, t)=0$. Then from Eqn 13, we see that the radial component $v_{\mathrm{r}}$ must be independent of $r$ and equal to $E / 2 / C$ :

$v_{\mathrm{r}}(r, l, t) \equiv v_{\mathrm{m}}(l, t)=\frac{E(l, t) / 2}{C}=\frac{\bar{E}(t) / 2}{C} \cdot f_{\mathrm{E}}(l)$,

where $f_{\mathrm{E}}(l)$ is a normalized function used to describe the spatial variations of transpiration rate along the leaf: $E(l, t)=\bar{E}(t) \cdot f_{\mathrm{E}}(l)$.

We further assume that $v_{1}(r, l, t)$ is independent of $r$ in the leaf vein, that is, $v_{1}(r, l, t)=v_{\mathrm{x}}(l, t)$. This is the case when water viscosity and vein wall rugosity can be neglected (Patankar 1980). Then from Eqn 13, we get (see the section on 'water velocity field' in Appendix II)

$\left.\frac{\partial v_{\mathrm{x}}}{\partial l}\right|_{l, t}=-\frac{2 v_{\mathrm{r}}\left(r_{\mathrm{x}}^{-}, l, t\right)}{r_{\mathrm{x}}}$,

where $v_{\mathrm{r}}\left(r_{\mathrm{x}}^{-}, l, t\right)$ is the radial velocity at $r=r_{\mathrm{x}}$ but still in the leaf xylem, which is denoted by $r_{\mathrm{x}}^{-}$. Equation 15 describes the fact that the divergence of the longitudinal water velocity along the vein is caused by a 'leakage' of this water into the mesophyll at $r=r_{\mathrm{x}}$. At any distance $l$ from the leaf base, the flux of liquid water leaving the xylem (through a halfcylindrical cross-sectional area $\pi r_{\mathrm{x}} \delta l$ ) must equal the flux of liquid water going into the (upper or lower) mesophyll (through a planar cross-sectional area $s_{\mathrm{X}} \delta l$ ). This is the continuity of the water flux at $r=r_{\mathrm{x}}$ :

$\pi r_{\mathrm{x}} \cdot v_{\mathrm{r}}\left(r_{\mathrm{x}}^{-}, l, t\right)=s_{\mathrm{x}} \cdot v_{\mathrm{r}}\left(r_{\mathrm{x}}^{+}, l, t\right)$

with $v_{\mathrm{r}}\left(r_{\mathrm{x}}^{+}, l, t\right)=v_{\mathrm{m}}(l, t)=E(l, t) / 2 C$. In other words, $v_{\mathrm{m}}(l, t)$ and $v_{\mathrm{r}}\left(r_{\mathrm{x}}^{-}, l, t\right)$ are different but proportional and the coefficient of proportionality is $s_{\mathrm{X}} / \pi r_{\mathrm{x}}$, which is easily understood by the conservation of mass.

At the vein tip $\left(l=l_{\max }\right)$, the longitudinal velocity is zero, that is, $v_{\mathrm{x}}\left(l_{\mathrm{max}}, t\right)=0$ and integration of Eqn 15 along the $l$-axis leads to

$v_{\mathrm{x}}(l, t)=v_{\mathrm{x} 0}(t) \cdot \int_{l}^{l_{\max }} f_{\mathrm{E}}\left(l^{\prime}\right) \cdot \frac{d l^{\prime}}{l_{\max }}$,

where $v_{\mathrm{x} 0}(t)$ is the velocity at the leaf base $(l=0)$ and is given by a simple mass balance over the entire vein:

$v_{\mathrm{x} 0}(t)=\frac{s_{\mathrm{x}} l_{\max }}{a_{\mathrm{x}}} \cdot \frac{\bar{E}(t)}{C}$.

Finally from Eqns 13 and 15, the radial velocity in the leaf veins is given by (see section on 'water velocity field' in Appendix II)

$v_{\mathrm{r}}(r, l, t)=\frac{s_{\mathrm{x}}}{2 \pi r_{\mathrm{x}}} \cdot \frac{\bar{E}(t)}{C} \cdot f_{\mathrm{E}}(l) \cdot \frac{r}{r_{\mathrm{x}}}$.

\section{Water isotope ratio in the xylem}

In the xylem, water isotopes are advected in the radial and longitudinal directions (with a velocity field given by Eqns 17 and 19), and are also diffused in both directions. The diffusivity in the longitudinal direction must be close to $D$, the tracer diffusivity in free liquid water, which is equal to $2.2 \times 10^{-9} \mathrm{~m}^{2} \mathrm{~s}^{-1}$ at $25^{\circ} \mathrm{C}$ for $\mathrm{H}_{2}{ }^{18} \mathrm{O}$ (Easteal et al. 1984). In contrast, the diffusivity in the radial direction is likely to be lower (by a factor $\kappa_{\mathrm{x}}<1$ ) because water isotopes must take a tortuous path to go from the vein vessels to the mesophyll. Then, if we denote by $R(r, l, t)$ the isotope ratio of leaf water at time $t$ and at a distance $r$ from the main axis of the leaf veins and a distance $l$ from the leaf base and with $R<<1$ (at natural abundance), the local continuity equation for the heavy isotope in the xylem is (see the section on 'water isotope ratio in the xylem' in Appendix II)

$\frac{\partial R}{\partial t}+\frac{1}{r} \frac{\partial r v_{\mathrm{r}} R}{\partial r}+\frac{\partial v_{1} R}{\partial l}=D\left\{\frac{\kappa_{\mathrm{x}}}{r} \frac{\partial}{\partial r}\left(r \frac{\partial R}{\partial r}\right)+\frac{\partial^{2} R}{\partial l^{2}}\right\}$,

which is a $2 \mathrm{D}$ advection-diffusion equation in polar coordinates with symmetry of revolution around the longitudinal axis. Equation 20 states that the rate of change of $R$ at a given location inside the vein (first term in Eqn 20) is caused by advection of the isotope in the radial and longitudinal directions (second and third terms, respectively) 
and by diffusion in the two directions (fourth and fifth terms, on the right-hand side). The boundary condition at $l=0$ is given by (Farquhar \& Gan 2003)

$$
v_{\mathrm{x} 0}(t) R_{\mathrm{s}}=v_{\mathrm{x} 0} R(r, 0, t)-\left.D \frac{\partial R}{\partial l}\right|_{r, 0, t},
$$

that is, the advected and diffused flux at $l=0$ in the leaf vein equals the incoming flux from the petiole with isotopic signature $R_{\mathrm{s}}$. In other words, not $R$ but the flux is continuous at $l=0$. This happens because complete mixing has been assumed in the petiole outside the leaf so that no isotopic gradient can exist for $l<0$ leading to this discontinuity in $R$ at $l=0$. Writing the same continuity of fluxes at $l=l_{\max }$ leads simply to

$$
\left.\frac{\partial R}{\partial l}\right|_{r, l_{\max }, t}=0
$$

because there is no outgoing flux in the longitudinal direction at the vein tip.

\section{Water isotope ratio in the mesophyll}

In the mesophyll, water isotopes are advected in the radial direction only, with velocity $v_{\mathrm{m}}(l, t)$ (Eqn 14), and diffuse in radial and longitudinal directions with an effective diffusivity $D_{\mathrm{m}}=\kappa_{\mathrm{m}} D\left(\mathrm{~m}^{2} \mathrm{~s}^{-1}\right)$. Then, with $R<<1$, the local continuity equation for water isotopes in this reservoir is (see section on 'water isotope ratio in the mesophyll' in Appendix II)

$$
\frac{\partial R}{\partial t}+v_{\mathrm{m}} \frac{\partial R}{\partial r}=D_{\mathrm{m}}\left\{\frac{\partial^{2} R}{\partial r^{2}}+\frac{\partial^{2} R}{\partial l^{2}}\right\} .
$$

Equation 22 is an advection-diffusion equation in two dimensions and states that the rate of change of $R$ at a given location in the mesophyll (first term in Eqn 22) is caused by advection of the isotope (second term, in the radial direction only) and diffusion (third and fourth term, on the righthand side). The boundary condition at the sites of evaporation $\left(r=r_{\mathrm{e}}=r_{\max }\right)$ is given by the continuity of the flux. As for total water, we assume no storage of water vapour isotopes in the mesophyll so that

$$
v_{\mathrm{r}}\left(r_{\mathrm{e}}, l, t\right) \cdot R\left(r_{\mathrm{e}}, l, t\right)-\left.D_{\mathrm{m}} \frac{\partial R}{\partial r}\right|_{r_{\mathrm{e}}, l, t}=\frac{E(l, t) / 2}{C} R_{\mathrm{E}}(l, t),
$$

where $R_{\mathrm{E}}(l, t)$ is the isotopic signature of the transpiration stream at time $t$ and distance $l$ from the leaf base and is given by Eqn 4 where $R_{\mathrm{e}}$ is now replaced by $R\left(r_{\mathrm{e}}, l, t\right)$. Equation 23 reduces exactly to eqn A21 of Farquhar \& Gan (2003), except that they state that it is valid at any distance $r$ and not only at $r=r_{\mathrm{e}}$. [Note that their radial velocity is equal to $2 v_{\mathrm{r}}\left(r_{\mathrm{e}}, l, t\right) / \kappa_{\mathrm{m}}$ in our notation]. In non-steady state when water isotopes can be stored in the mesophyll, this is not the case anymore and Eqn 23 is only valid at $r=r_{\mathrm{e}}$. Combining Eqn 4 with Eqn 23 and replacing $v_{\mathrm{r}}$ by Eqn 14 gives
$\left.D_{\mathrm{m}} \frac{\partial R}{\partial r}\right|_{r_{\mathrm{e}}, l, t}+\frac{v_{\mathrm{m}}(l, t)}{(1-h)} \cdot \frac{h^{\prime}}{\alpha_{\mathrm{k}} \alpha^{+}} R\left(r_{\mathrm{e}}, l, t\right)=\frac{v_{\mathrm{m}}(l, t)}{(1-h)} \cdot \frac{h R_{\mathrm{v}}}{\alpha_{\mathrm{k}}}$.

The boundary conditions at $l=0$ and $l=l_{\max }$ are also given by the continuity of the flux, which reduces to

$$
\left.\frac{\partial R}{\partial l}\right|_{r, 0, t}=\left.\frac{\partial R}{\partial l}\right|_{r, l_{\max }, t}=0
$$

because there is no advection in the longitudinal direction within the mesophyll. Finally, as for total water, the flux of liquid water leaving the xylem at any distance $l$ from the leaf base must equal the flux of liquid water going into the mesophyll. This is the continuity of the flux at $r=r_{\mathrm{x}}$ and any distance $l$ from the leaf base, and it leads to

$$
\begin{aligned}
\pi r_{\mathrm{x}} & \cdot\left(v\left(r_{\mathrm{x}}^{-}, l, t\right) R\left(r_{\mathrm{x}}^{-}, l, t\right)-\left.D_{\mathrm{x}} \frac{\partial R}{\partial r}\right|_{r_{\mathrm{x}}^{-}, l, t}\right) \\
= & s_{\mathrm{x}} \cdot\left(v_{\mathrm{m}}(l, t) R\left(r_{\mathrm{x}}^{+}, l, t\right)-\left.D_{\mathrm{m}} \frac{\partial R}{\partial r}\right|_{r_{\mathrm{x}}^{+}, l, t}\right),
\end{aligned}
$$

where $D_{\mathrm{x}}=\kappa_{\mathrm{x}} D$. If $R$ varies continuously at the xylem/ mesophyll boundary, then $R\left(r_{\mathrm{x}}^{-}, l, t\right)=R\left(r_{\mathrm{x}}^{+}, l, t\right)$ and Eqn 25 states that the gradients at both sides of this boundary are proportional by a factor $S_{\mathrm{x}} / \pi r_{\mathrm{x}} \cdot \kappa_{\mathrm{m}} / \kappa_{\mathrm{x}}$.

\section{Numerical solution}

Our leaf water isotope enrichment model is solved numerically using the control volume method of Patankar (1980). The leaf longitudinal xylem and the leaf mesophyll are both decomposed in $20 \times 40$ cells (or control volumes) with decreasing size towards leaf tip. On each cell wall, the velocity field is first computed according to Eqns 14, 17 and 19. For that, a given mean evaporation rate $\bar{E}$, assumed constant over a period $(t, t+d t)$ but spatially variable with shape $f_{\mathrm{E}}(l)$, is required. The shape of $f_{\mathrm{E}}(l)$ is actually deduced from prescribed shapes of stomatal conductance and leaf temperature $\left[f_{\mathrm{g}}(l)\right.$ and $f_{\mathrm{T}}(l)$, respectively], and using Eqn 1 and $1 / g_{\mathrm{t}}=1 / g_{\mathrm{s}}+1 / g_{\mathrm{b}}$. Then the two advectiondiffusion equations (Eqns 20 and 22) are integrated over each control volume and solved numerically. Boundary conditions (Eqns 21a, 21b, 24a, 24b and 25) are used for control volumes at the periphery. In Eqn $24 \mathrm{a}, E /(1-h)$ must be replaced by $g_{\mathrm{t}} w_{\mathrm{i}}$ in case $h=1$ (i.e. $E=0$ ) but $g_{\mathrm{t}} \neq 0$. If the initial condition $R(r, l, t)$ is known, these integral equations form a closed set of linear equations that can be solved numerically to calculate $R(r, l, t+d t)$ at any point in the leaf. In practice, we used $d t=300 \mathrm{~s}$ and solved the equations iteratively for over longer time intervals.

\section{RESULTS AND DISCUSSION}

\section{Model parameterization}

A list of model parameters is given in Table 1, together with typical values and the expected ranges for mature maize leaves. We distinguish four types of parameters: (1) 


\begin{tabular}{|c|c|c|}
\hline Parameter & Typical value (maize leaf) & Expected range \\
\hline \multicolumn{3}{|l|}{ Leaf dimensions $\mathrm{s}^{\mathrm{a}}$} \\
\hline$l_{\max }(\mathrm{cm})$ & 50 & $25-125$ \\
\hline$s_{\mathrm{x}}(\mathrm{mm})$ & 1.7 & $0.5-3.5$ \\
\hline$a_{\mathrm{x}}\left(\mu \mathrm{m}^{2}\right)$ & 3400 & $2000-4000$ \\
\hline$r_{\mathrm{m}}(\mu \mathrm{m})$ & 120 & $50-300$ \\
\hline \multicolumn{3}{|c|}{ Tortuosity and effective diffusivity factors ${ }^{\mathrm{b}}$} \\
\hline$\kappa_{\mathrm{m}}\left(\times 10^{-3}\right)$ & 5 & $1-100$ \\
\hline$\kappa_{\mathrm{x}}\left(\times 10^{-3}\right)$ & 10 & $10-1000$ \\
\hline \multicolumn{3}{|c|}{ Gas-exchange parameters ${ }^{\mathrm{c}}$} \\
\hline $\bar{E}\left(\mathrm{mmol} \mathrm{m}^{-2} \mathrm{~s}^{-1}\right)$ & 4 & $0-10$ \\
\hline$g_{\mathrm{b}}\left(\mathrm{mol} \mathrm{m}^{-2} \mathrm{~s}^{-1}\right)$ & 0.5 & $0.1-2.5$ \\
\hline$P_{\text {air }}(\mathrm{kPa})$ & 101.3 & 101.3 \\
\hline$D_{\text {air }}(\mathrm{kPa})$ & 2 & $0-5$ \\
\hline$T_{\text {air }}\left({ }^{\circ} \mathrm{C}\right)$ & 28 & $0-45$ \\
\hline $\bar{T}_{\text {leaf }}\left({ }^{\circ} \mathrm{C}\right)$ & 30 & $0-45$ \\
\hline \multicolumn{3}{|l|}{ Isotopic parameters $^{\mathrm{d}}$} \\
\hline$\Delta_{\mathrm{v}}(\% \mathrm{o})$ & $-10[-80]$ & -20 to $0[-160$ to 0$]$ \\
\hline$\varepsilon_{\mathrm{k}, 0}(\% \mathrm{o})$ & $28.5[25.1]$ & 28.5 to 31.9 [16.4 to 25.1$]$ \\
\hline
\end{tabular}

Table 1. List of model parameters, their typical values and the expected ranges for a mature corn leaf

${ }^{\mathrm{a}}$ In our model, the interveinal distance $s_{\mathrm{x}}$ is the distance between lateral veins. Intermediate veins are implicitly accounted for in the model, through $a_{\mathrm{x}}$ (which is the total cross-sectional area of all conducting vessels between two adjacent lateral veins) and by assuming that the flow of water entering the mesophyll is uniform along the plane $r=r_{\mathrm{x}}$ (Fig. 3). Details of the calculation for $a_{\mathrm{x}}$ can be found in Gan et al. (2003).

${ }^{\mathrm{b}} \mathrm{A}$ value of 1000 corresponds to zero tortuosity and leads to the tracer diffusivity in water. ${ }^{\mathrm{c}}$ We can give either a mean transpiration rate $\bar{E}$ or a mean stomatal conductance $\bar{g}_{\mathrm{s}}$, because they are linked through Eqn 1. In the latter case, $\bar{g}_{\mathrm{s}}$ is expected to have a typical value of $0.25 \mathrm{~mol} \mathrm{~m}^{-2} \mathrm{~s}^{-1}$ with an expected range of $0.01-0.5 \mathrm{~mol} \mathrm{~m}^{-2} \mathrm{~s}^{-1}$.

${ }^{\mathrm{d}}$ Values are given for oxygen-18. Values for deuterium are in brackets.

geometrical parameters related to leaf dimensions $\left(l_{\max }, s_{\mathrm{x}}\right.$, $\left.a_{\mathrm{x}}, r_{\mathrm{m}}\right),(2)$ dynamical parameters that account for the tortuosity of the water path and the type of diffusion in the leaf xylem $\left(\kappa_{\mathrm{x}}\right)$ and mesophyll $\left(\kappa_{\mathrm{m}}\right)$, (3) leaf gas-exchange parameters $\left(\bar{E}, \mathrm{~g}_{\mathrm{b}}\right.$, air pressure $P_{\mathrm{air}}$, air vapour pressure deficit $D_{\text {air }}$, air temperature $T_{\text {air }}$, mean leaf temperature $\bar{T}_{\text {leaf }}$ ) and (4) isotopic parameters $\left(\Delta_{\mathrm{v}}=R_{\mathrm{v}} / R_{\mathrm{s}}-1\right.$ and $\left.\varepsilon_{\mathrm{k}, 0}\right)$. A mean stomatal conductance is then computed from these parameters using Eqn 1 with $1 / g_{\mathrm{t}}=1 / g_{\mathrm{s}}+1 / g_{\mathrm{b}}$. If stomatal conductance and/or leaf temperature are non-uniform along the leaf blade, additional parameters are needed to parameterize the normalized functions $f_{\mathrm{g}}$ and $f_{\mathrm{T}}$. These normalized functions are expected to have one maximum, quite smooth variations along the leaf blade and non-zero minima. We can therefore approximate $f_{\mathrm{g}}$ and $f_{\mathrm{T}}$ by two Gaussian curves with a positive minimum threshold $f_{\min }$, a distance $l_{\text {opt }}$ where the maximum is reached and an SD around that maximum $\sigma_{\mathrm{l}}$. The maximum $f_{\max }$ is then computed by normalization $\left(\int_{0}^{1} f(l) d l=1\right)$.

To illustrate this, we plotted in Fig. 4 a three-dimensional representation of the isotope composition of leaf water $R(r, l)$ at steady state $(t \rightarrow \infty)$ with typical parameter values shown in Table 1 and assuming realistic Gaussian variations of both $g_{\mathrm{s}}\left(l_{\mathrm{opt}} / l_{\max }=0.7 ; \sigma_{\mathrm{l}}=0.3 ; f_{\min }=0.85\right)$ and $T_{\text {leaf }}\left(l_{\mathrm{opt}} /\right.$ $\left.l_{\max }=0.3 ; \sigma_{1}=0.25 ; f_{\min }=0.85\right)$. We have $r_{\mathrm{x}} / r_{\max } \approx 0.2$ so that cells at $r / r_{\max }<0.2$ are in the leaf longitudinal xylem and cells at $r / r_{\max }>0.2$ are in the mesophyll. This plot shows a general enrichment from $l=0$ to $l=l_{\max }$ and from $r=0$ to $r=r_{\text {max }}$. The radial variations in both reservoirs depend strongly on the values of $\kappa_{\mathrm{x}}$ and $\kappa_{\mathrm{m}}$, and the local maximum enrichment at $r / r_{\max }=1$ and $l / l_{\max }=0.4-0.5$ is due to the maximum in leaf temperature. The influence of varying stomatal conductance is much smaller compared to nonuniform $T_{\text {leaf }}$, and is not visible in Fig. 4.

As explained in the 'Model description' section, we assume that the longitudinal velocity in the leaf veins is independent of $r$, that is, $v_{1}(r, l, t)=v_{\mathrm{x}}(l, t)$. We also tested two other shapes of the radial profile of $v_{1}$ inside the veins: a linear decrease and a parabolic decrease from $r=0$ to $r=r_{\mathrm{x}}$. In both cases, the carpet shown in Fig. 4 was not greatly affected (not shown), indicating that the choice of the radial profile of $v_{1}$ inside the veins is not crucial in our model. In the following discussion, a constant radial profile for $v_{1}$ is used and a sensitivity analysis of the model to different parameters is presented.

\section{Model sensitivity to $\kappa_{\mathrm{m}}$ and $\kappa_{\mathrm{x}}$}

We first tested the role played by $\kappa_{\mathrm{x}}$ and $\kappa_{\mathrm{m}}$ on the progressive enrichment of leaf water corresponding to different steady-state experiments taken from the literature (Helliker \& Ehleringer 2000; Gan et al. 2003; Affek et al. 2006). [This exercise is very similar to the one in Farquhar \& Gan (2003) who looked at the sensitivity of their model to different values of the Péclet numbers]. Apart from the experiment called 'Helliker' that was performed on 

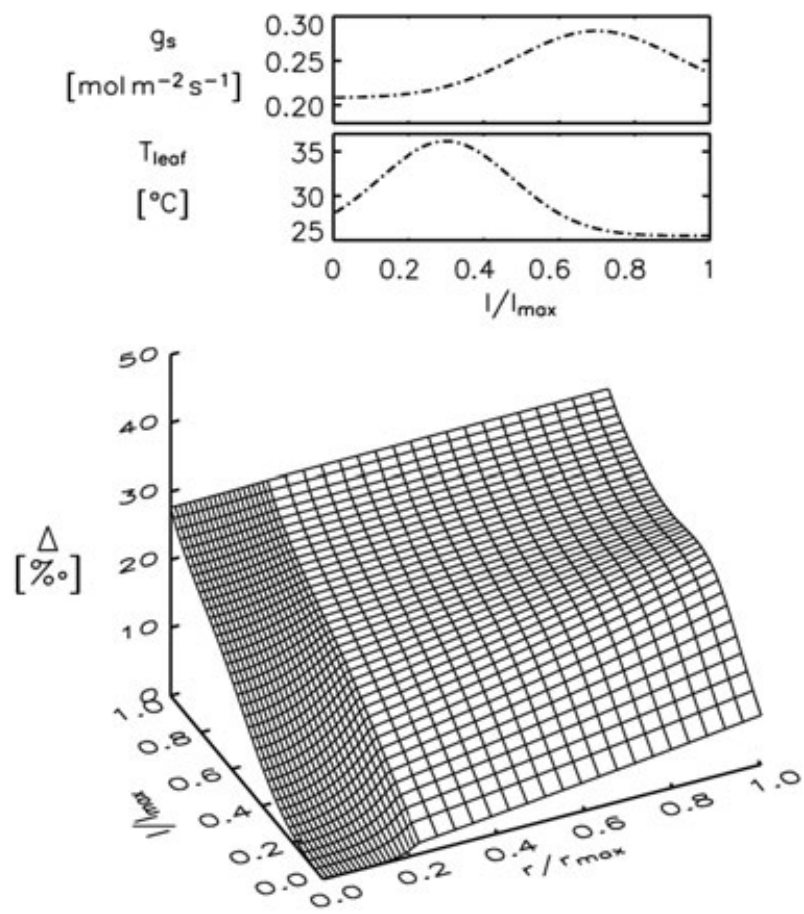

Figure 4. Three-dimensional representation of the isotope ratio of leaf water at steady state, expressed relative to source water: $\Delta(r, l)=R(r, l) / R_{\mathrm{s}}-1$. This plot has been obtained with the parameter values in Table 1 and assuming Gaussian variations of both $g_{\mathrm{s}}$ and $T_{\text {leaf }}$, as shown in the top panel of the figure (see text for details).

Miscanthus sinensis leaves (Helliker \& Ehleringer 2000), all other experiments were using Zea mays (corn) leaves of different lengths and subjected to different environmental conditions. Details of these experiments are listed in Table 2. In some of these studies, the 'bulk' leaf water enrichment $\left(\Delta_{\mathrm{lw}}=R_{\mathrm{lw}} / R_{\mathrm{s}}-1\right)$ was measured, without any distinction between mesophyll and xylem water. The latter can be expressed in terms of enrichment in the vein $\Delta_{\mathrm{x}}\left(=R_{\mathrm{x}} / R_{\mathrm{s}}-1\right)$ and in the mesophyll $\Delta_{\mathrm{m}}\left(=R_{\mathrm{m}} / R_{\mathrm{s}}-1\right)$ (Gan et al. 2003):

$$
\Delta_{\mathrm{lw}}(l)=\phi_{\mathrm{x}} \cdot \Delta_{\mathrm{x}}(l)+\left(1-\phi_{\mathrm{x}}\right) \cdot \Delta_{\mathrm{m}}(l),
$$

where $\phi_{\mathrm{x}}$ denotes the volume fraction of the leaf occupied by veins. The latter is directly deduced from the leaf dimensions in Table 1: $\phi_{\mathrm{x}}=a_{\mathrm{x}} /\left(a_{\mathrm{x}}+2 s_{\mathrm{x}} r_{\mathrm{m}}\right)$. To be consistent with the leaf geometry used, and in contrast to Gan et al. (2003), the volume fraction of the ground tissues that surround the veins is not included in $\phi_{\mathrm{x}}$ and these tissues are rather included in the mesophyll. Our value of $\phi_{\mathrm{x}}(0.01)$ is therefore much smaller than the value that Gan et al. (2003) obtained by gravimetric measurements $(0.43)$. In any case, the sensitivity of $\Delta_{\mathrm{lw}}(l)$ to $\phi_{\mathrm{x}}$ is quite small and different values of $\phi_{\mathrm{x}}$ should not influence the major outcomes of our study. Indeed if, at any distance from the leaf base, $\Delta_{\mathrm{x}}(l)$ is less enriched than $\Delta_{\mathrm{m}}(l)$ by, for example, $5 \%$ (which is large in the light of Fig. 4) then changing $\phi_{\mathrm{x}}$ from 0.01 to 0.43 would lead to a reduction of $\Delta_{\mathrm{lw}}(l)$ by about $(0.43-$ $0.01) \times 5 \%=2 \%$, that is, an order of magnitude smaller than the total enrichment of $\Delta_{\mathrm{lw}}(l)$ along the leaf blade (see Fig. 4).

In the top panel of Fig. 5, we compared the measured progressive enrichment for these different experiments with the values obtained with the present model and our standard values of $\kappa_{\mathrm{x}}$ and $\kappa_{\mathrm{m}}\left(\kappa_{\mathrm{x}}=0.010\right.$ and $\kappa_{\mathrm{m}}=0.005$; see Table 1). We also plotted the values predicted by the model of Farquhar \& Gan (2003) and by the present model, if we assume complete mixing in the radial direction inside the vein $\left(\kappa_{\mathrm{x}}=10^{9} \rightarrow \infty\right)$ and no longitudinal diffusion in the mesophyll $\left(D_{\mathrm{lm}} / D=0\right)$, in order to check that they predict the same results. We can see that the model curves agree reasonably well with the experimental data from the four first data sets. In the experiment called 'Affek', they tend to overestimate the isotopic enrichment for $l>0.4 l_{\max }$. Moreover, in all experiments, both models predict a higher isotopic gradient at the leaf tip compared to the measurements, as was already observed by Gan et al. (2003) with the model of Farquhar \& Gan (2003). We show here that the inclusion of longitudinal diffusion in the mesophyll does not reduce this isotopic gradient at the leaf tip, contrary to what has been suggested (Farquhar \& Gan 2003; Gan et al. 2003).

In the second panel of the same figure, we plotted $\Delta_{\mathrm{lw}}(l)$ values obtained with the present model and different values of $\kappa_{\mathrm{m}}(0.025,0.005$ and 0.001$)$, while assigning all other parameters to the values listed in Table 2. For all experiments, we can see that the model is very sensitive to $\kappa_{\mathrm{m}}$ and predicts a higher enrichment for higher values of the parameter (or a lower Péclet number $\wp_{\mathrm{m}}$ ). For example, the agreement between model and measurements in the 'Affek' experiments is improved if we take $\kappa_{\mathrm{m}}=0.001$. Using a fivefold smaller $\kappa_{\mathrm{m}}$ for the 'Affek' experiment is not justified if

Table 2. List of model parameters used for the different steady-state experiments shown in Fig. 5

\begin{tabular}{lllllc}
\hline & $\begin{array}{l}\text { Gan } \\
(x y l e m)\end{array}$ & $\begin{array}{l}\text { Gan } \\
(\text { low })\end{array}$ & $\begin{array}{l}\text { Gan } \\
(\text { high })\end{array}$ & Helliker & Affek \\
\hline Leaf dimensions & & & & & \\
$\quad l_{\max }(\mathrm{cm})$ & 50 & 75 & 75 & 12 & 80 \\
$\quad s_{\mathrm{x}}(\mathrm{mm})$ & 1.7 & 1.7 & 1.7 & 0.12 & 1.7 \\
Gas-exchange & & & & & \\
parameters & & & & & \\
$\bar{E}\left(\mathrm{mmol} \mathrm{m}^{-2} \mathrm{~s}^{-1}\right)$ & 7 & 9 & 5 & $3.2^{\mathrm{a}}$ & 1.9 \\
$g_{\mathrm{b}}\left(\mathrm{mol} \mathrm{m}^{-2} \mathrm{~s}^{-1}\right)$ & 0.52 & 0.52 & 0.52 & 0.50 & 0.50 \\
$D_{\text {air }}(\mathrm{kPa})$ & 2.12 & 2.70 & 1.07 & 2.06 & 1.43 \\
$T_{\text {air }}\left({ }^{\circ} \mathrm{C}\right)$ & 30 & 30 & 30 & 25 & 21.7 \\
$\quad \bar{T}_{\text {leaf }}\left({ }^{\circ} \mathrm{C}\right)$ & 34 & 34 & 34 & 27 & 22.2 \\
Isotopic parameters & & & & & \\
$\Delta_{\mathrm{v}}(\%)$ & -4.3 & -4.3 & -4.3 & -14.7 & -9.7 \\
\hline
\end{tabular}

The transpiration rate was not measured in this experiment but, according to the Craig-Gordon values reported by Helliker \& Ehleringer (2000), we have $g_{\mathrm{b}} / g_{\mathrm{s}}=0.35$ and with, $g_{\mathrm{b}}=0.5 \mathrm{~mol}$ $\mathrm{m}^{-2} \mathrm{~s}^{-1}$ and the measured value of $\bar{T}_{\text {leaf }}$, we end up with a transpiration rate of $3.2 \mathrm{mmol} \mathrm{m}^{-2} \mathrm{~s}^{-1}$.

All other model parameters are as in Table 1 . 


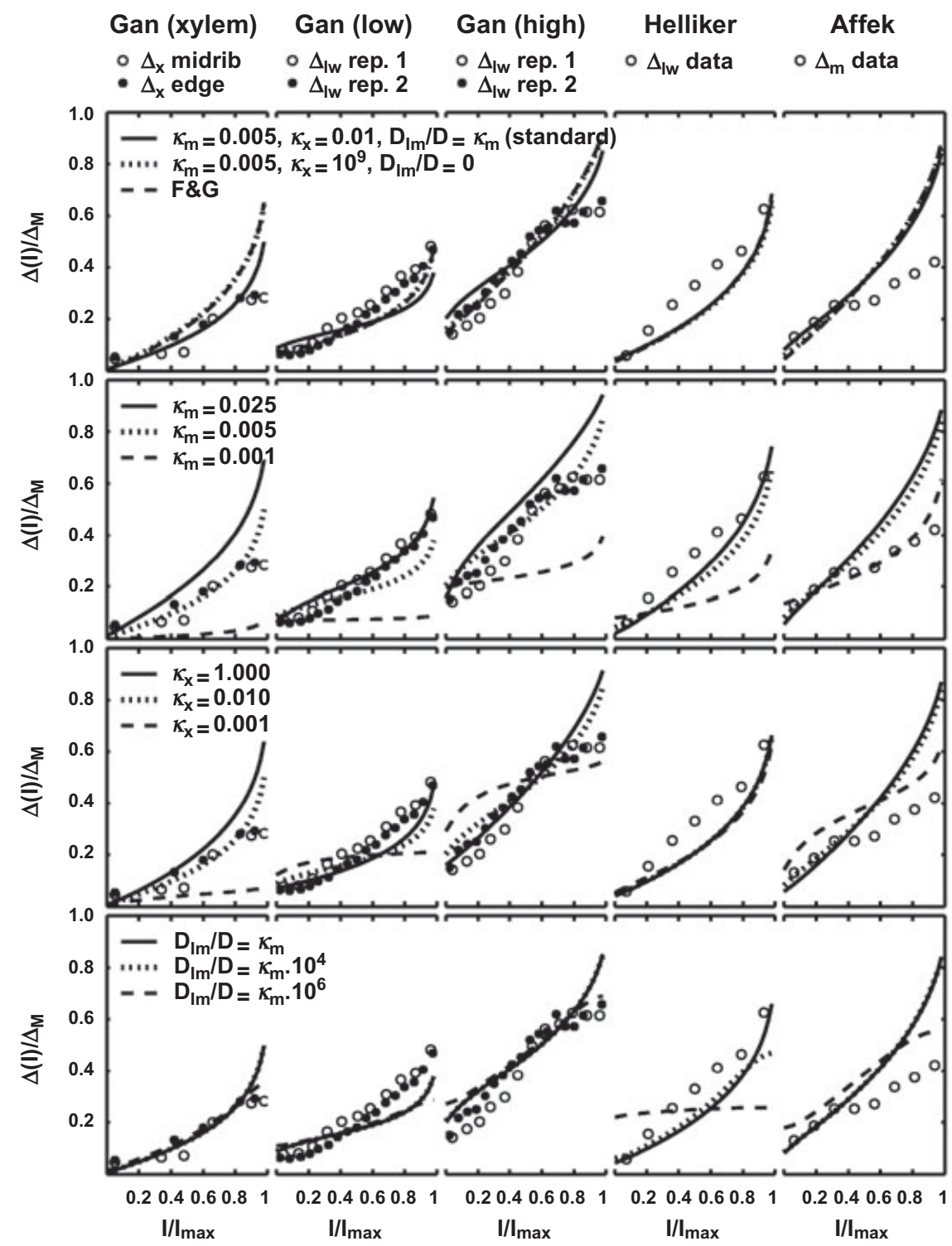

Figure 5. Progressive enrichment patterns corresponding to different steady-state experiments taken from the literature: 'Gan (xylem)' focuses on the spatial patterns of $\Delta_{\mathrm{x}}(l)$ alone in a corn leaf (Gan et al. 2003); 'Gan (low)' and 'Gan (high)' show spatial patterns of $\Delta_{\mathrm{lw}}(l)$ in corn leaves (two replicates) for low and high humidity conditions (Gan et al. 2003); 'Helliker' shows spatial patterns of $\Delta_{\mathrm{lw}}(l)$ in a Miscanthus sinensis leaf (Helliker \& Ehleringer 2000) and 'Affek' shows spatial patterns of $\Delta_{\mathrm{m}}(l)$ in a corn leaf in the field (Affek et al. 2006). Details of these experiment are listed in Table 2. Experimental values are plotted with open or filled circles (depending on the number of replicates) and modelled values are plotted with curves of different styles (depending on the values of $\kappa_{\mathrm{x}}$ and $\kappa_{\mathrm{m}}$, or the ratio of the diffusivity in the longitudinal direction in the mesophyll, $D_{\mathrm{lm}}$, to $D$ in case diffusion is not isotropic in this reservoir). 'F\&G' holds for the steady-state model of Farquhar \& Gan (2003).

we suppose that $\kappa_{\mathrm{m}}$ is species-specific only. However, our model does not take into account changes in leaf water volume in the mesophyll and this should result in a reduced $\kappa_{\mathrm{m}}$. More importantly, we know that leaf water can take simultaneously different pathways in the mesophyll, each pathway having a specific tortuosity (Barbour \& Farquhar 2003). It is possible that the relative importance of these different pathways depends on genotypes and/or growth and environmental conditions. This questions the use of one single value of $\kappa_{\mathrm{m}}$ for the same species but different plants or environmental conditions.
In the third panel of Fig. 5, we repeated the same exercise but now with different values of $\kappa_{\mathrm{x}}(1.000,0.010$ and 0.001$)$. It shows that the model can be quite sensitive to this parameter, but only when $\kappa_{\mathrm{x}}<0.010$. Indeed, above this threshold the model predicts nearly the same results as with $\kappa_{\mathrm{x}}=10^{9} \rightarrow \infty$ (shown in the top panel). Because values of $\kappa_{\mathrm{x}}$ below 0.010 are probably too unrealistic, we conclude that a precise determination of the value of $\kappa_{\mathrm{x}}$ is not crucial.

In the last panel of the same figure, we tested the effect of an anisotropic diffusion in the mesophyll. For that we used different values $\left(1,10^{4}\right.$ and $\left.10^{6}\right)$ of the ratio of the 
longitudinal diffusivity (noted $D_{\operatorname{lm}}$ for this purpose) to the radial diffusivity $\kappa_{\mathrm{m}} D$. An enhanced longitudinal diffusion in the mesophyll by a factor $10^{6}$ is needed to start seeing a reduction in the isotopic gradient at the leaf tip. The same conclusion would be obtained if we increased longitudinal diffusion in the leaf vein, as was done by Farquhar \& Gan (2003) and Gan et al. (2003) with their steady-state model. This means that longitudinal diffusion in the liquid phase (in xylem and/or mesophyll) cannot explain these lowerthan-predicted isotopic gradients and that other processes must be involved, which take away heavy water isotopes from the leaf tip.

Diffusion of water vapour in the mesophyll intercellular air space is not accounted for in our model and the tracer diffusivity in the vapour phase is $10^{5}$ higher than $D$. However, we do not think that water vapour diffusion could lead to a much reduced isotopic gradient at the leaf tip. This is because the saturating water vapour molar concentration in the air is $10^{4}-10^{5}$ smaller than $C$, and so the product of diffusivity and molar concentration (the diffusion flux) is little different for the two phases. In contrast, we think that tapering of the leaf blade could be a plausible explanation. Such effect is difficult to incorporate in our model because we consider only one single vein. However, we can always assume variations of the interveinal distance $s_{\mathrm{X}}$ along the leaf. Some preliminary tests show that, when we reduce $s_{\mathrm{x}}$ from its nominal value to zero between $0.6 l_{\max }$ and $l_{\max }$, the model predicts a much-reduced isotopic gradient at the leaf tip (not shown). Lower isotopic gradients at the leaf tip might also be due to a non-uniform transpiration rate along the leaf, with a smaller rate (and therefore a smaller enrichment) at the leaf tip. The effect of spatially varying gasexchange parameters is analysed next.

\section{Model sensitivity to spatial variations of gas-exchange parameters $\left(g_{\mathrm{s}}, T_{\text {leaf }}\right)$}

In the 'Affek' experiment, variations of stomatal conductance and leaf temperature along the leaf blade were measured (Affek et al.2006) and can be used to test the effect of a non-uniform transpiration rate on the shape of $\Delta_{\mathrm{m}}(l)$. This effect is shown in the top panel of Fig. 6 where we plotted the measured isotopic enrichment of mesophyll water $\Delta_{\mathrm{m}}(l)$ together with the values of $\Delta_{\mathrm{m}}(l)$ predicted by the present model for a uniform transpiration rate (equal to the mean value $\bar{E}$, as in Fig. 5) or two non-uniform transpiration rates (one directly deduced from the measured variations in $g_{\mathrm{s}}$ and $T_{\text {leaf }}$ and another one with fitted profiles of $g_{\mathrm{s}}$ and $T_{\text {leaf }}$ ). Measured and fitted variations in $g_{\mathrm{s}}, T_{\text {leaf }}$ and $E$ are also shown in Fig. 6. All model parameters were taken from Table 2, except the tortuosity factor $\kappa_{\mathrm{m}}$ that was reduced from 0.005 to 0.0015 . This reduction of $\kappa_{\mathrm{m}}$ is needed to have a reasonable agreement between measured and modelled curves, even with uniform $g_{\mathrm{s}}$ and $T_{\text {leaf }}$, as shown in Fig. 5. If we further take into account the measured variations in $g_{\mathrm{s}}$ and $T_{\text {leaf }}$ along the leaf, the isotopic gradient at the leaf tip is slightly reduced and the agreement with the measurements is improved in this region. However, some discrepancies greater than $5 \%$ still remain.

Gas-exchange measurements were performed between 11 and $13 \mathrm{~h}$ (Affek et al. 2006), but necessarily before the leaf was cut into segments for the isotopic measurements. This temporal shift results in different environmental conditions (including solar zenith angle) for the different data types and might explain why we observe a local maximum in $\Delta_{\mathrm{m}}$ at $l / l_{\max }=0.35$ while the local maximum in $g_{\mathrm{s}}$ and $T_{\text {leaf }}$ is measured at $l / l_{\max }=0.45$ and $l / l_{\max }=0.55$, respectively. These differences in the local maxima could also involve some other processes because leaf water isotopic enrichment should not necessarily exhibit the same spatial patterns as $g_{\mathrm{s}}$ or $T_{\text {leaf }}$ Still, the perfect adequacy between $g_{\mathrm{s}}, T_{\text {leaf }}$ and $\Delta_{\mathrm{m}}$ measurements can be questioned. Bearing this in mind, we then tried to 'adjust' the spatial patterns of $g_{\mathrm{s}}$ and $T_{\text {leaf }}$ in order to reduce the discrepancies on $\Delta_{\mathrm{m}}$ between the model and the measurements. Such a fit is not unique and several profiles of $g_{\mathrm{s}}$ and $T_{\text {leaf }}$ can lead to the same result. The fitted profiles shown in Fig. 6 have been obtained by shifting the local maximum in $g_{\mathrm{s}}$ from 0.45 to $0.35 l_{\max }$ and then fitting the $T_{\text {leaf }}$ profile. An increase of about $2{ }^{\circ} \mathrm{C}$ at $0.35 l_{\max }$ was still necessary to reproduce the local maximum in $\Delta_{\mathrm{m}}$. More importantly, cooler leaf temperatures by about $5{ }^{\circ} \mathrm{C}$ were needed to reproduce the observed isotopic

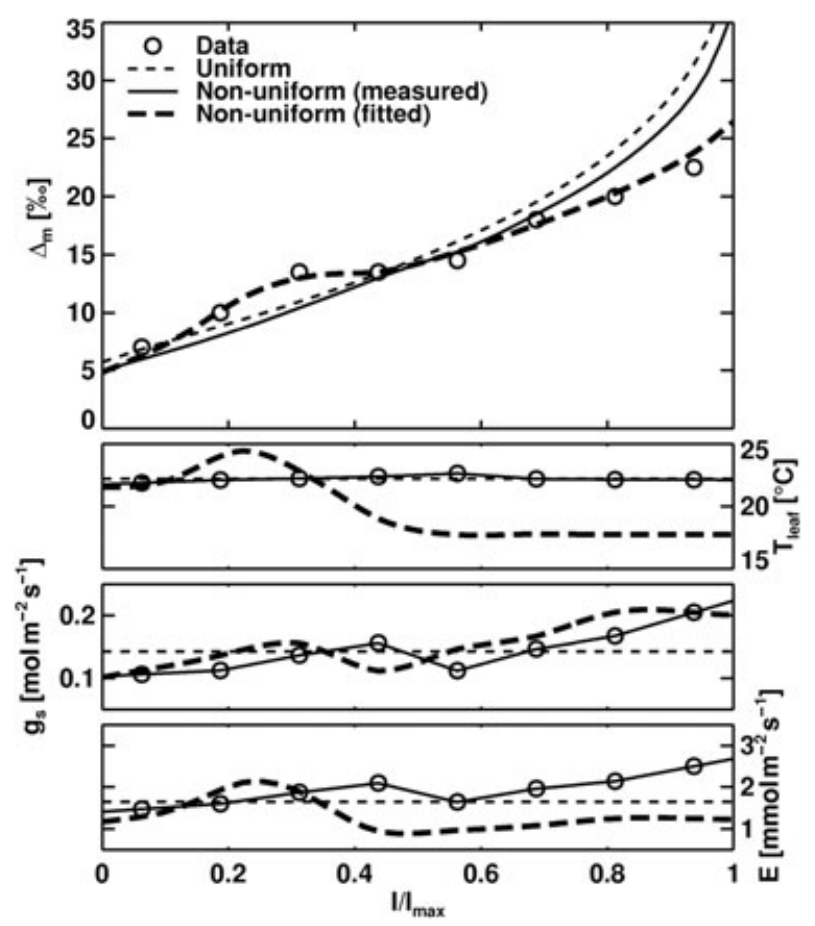

Figure 6. Measured and modelled steady-state progressive isotopic enrichment of mesophyll water $\Delta_{\mathrm{m}}(l)$ along a corn leaf (top panel) and the corresponding measured and modelled variations of leaf temperature $T_{\text {leaf }}(l)$, stomatal conductance $g_{\mathrm{s}}(l)$ and transpiration rate $E(l)$. The experimental data are taken from Affek et al. (2006), except the leaf temperature that has been provided independently by D. Yakir (personal communication). 
enrichment at the leaf tip. This fitted leaf temperature profile is quite unrealistic because, in the field around midday, the vegetation is expected to be well coupled to the atmosphere so that $T_{\text {leaf }}$ should not be greatly different from $T_{\text {air }}$ (less than $2{ }^{\circ} \mathrm{C}$ ). However, the $g_{\text {s }}$ profile shown in Fig. 6 is not really fitted but only shifted towards the base of the leaf. This results in larger values of $g_{\mathrm{s}}$ for $l / l_{\max }=0.6-0.9$, compared to the measurements or the mean value, which are then artificially compensated for with the $T_{\text {leaf }}$ profile by cooling the temperatures in this region of the leaf by about $5{ }^{\circ} \mathrm{C}$. Again, if we account for tapering of the leaf blade (by reducing $s_{\mathrm{x}}$ at the leaf tip) then the adjustment becomes much closer $\left(<2{ }^{\circ} \mathrm{C}\right)$ to the measurements (not shown).

Overall, if we take into account all possible uncertainties in the measurements and model parameters, we believe that our model can explain the observed $\Delta_{\mathrm{m}}$ patterns along the leaf blade, at least up to about $0.8-0.9 l_{\max }$. Closer to the leaf tip, we consider that our model is less well adapted. Indeed, at such distance from the leaf base, effects such as tapering of the leaf blade (i.e. variations in $s_{\mathrm{x}}$ ) but also tapering of the leaf veins (i.e. variations in $r_{\mathrm{x}}$ ) and changes in leaf water volume (i.e. variations of $r_{\max }$ ) are quite important and difficult to account for in the model, which complicates comparison of our model with measurements in this region of the leaf.

\section{Model sensitivity to leaf dimensions ( $\left.I_{\max }, s_{\mathrm{x}}\right)$}

As mentioned in the Introduction, Helliker \& Ehleringer (2000) observed differences of steady-state bulk leaf water enrichment $\bar{\Delta}_{\mathrm{lw}}$ between $\mathrm{C}_{3}$ and $\mathrm{C}_{4}$ plants and proposed that leaf length $l_{\max }$ and interveinal distance $s_{\mathrm{x}}$ were responsible for these differences. Our model, which explicitly accounts for these two parameters, can be used to explore the possible link between $\bar{\Delta}_{\mathrm{lw}}$ and leaf geometry. For that we used $\left\{s_{\mathrm{x}}, l_{\max }\right\}$ values measured by Helliker \& Ehleringer (2000) for all species they studied and the values in Table 2 for the other parameters and computed bulk steady-state leaf water enrichment according to our model. Contrary to what Helliker \& Ehleringer (2000) proposed, our modelled values did not exhibit any measurable correlation with both $s_{\mathrm{x}}$ and $l_{\max }\left(\mathrm{SD}\right.$ on $\bar{\Delta}_{\mathrm{lw}}$ values less than $0.03 \%$ between all species; there is no correlation with $l_{\max }$ but a very small correlation with $s_{\mathrm{x}}$ ). This absence of a noticeable correlation is actually easy to understand and is inherent to our model. In steady state, and if we assume a uniform transpiration rate, that is, $f_{\mathrm{E}}(l)=1$, the deviation of bulk leaf water from the Craig-Gordon value is equal to (see section on 'bulk steady-state isotope ratios' in Appendix II)

$1-\frac{\bar{\Delta}_{\mathrm{lw}}}{\Delta_{\mathrm{C}}}=\phi_{\mathrm{x}} \cdot\left(1-f_{\mathrm{xx}} e^{-\wp_{\mathrm{m}}}\right)+\left(1-\phi_{\mathrm{x}}\right) \cdot\left(1-f_{\mathrm{em}}\right)$,

where

$\left\{\begin{array}{l}f_{\mathrm{em}}=\frac{1-e^{-\wp_{\mathrm{m}}}}{\wp_{\mathrm{m}}} \\ f_{\mathrm{xx}}=\frac{1-e^{-\wp_{\mathrm{x}}}}{\wp_{\mathrm{x}}}\end{array}\right.$

and $\wp_{\mathrm{x}}$ is the radial Péclet number in the vein:

$\wp_{\mathrm{x}} \equiv \frac{\bar{v}\left(r_{\mathrm{x}}^{-}\right) r_{\mathrm{x}}}{2 D_{\mathrm{x}}}=\frac{\bar{E}}{C D} \cdot \frac{s_{\mathrm{x}}}{4 \pi \kappa_{\mathrm{x}}}$.

Equation 27 shows that, in our model, $\bar{\Delta}_{\mathrm{lw}}$ is completely independent of $l_{\max }$ and depends on $s_{\mathrm{x}}$ through $\phi_{\mathrm{x}}$ and $f_{\mathrm{xx}}$ only. But because $\phi_{\mathrm{x}}$ is close to zero (i.e. $a_{\mathrm{x}}<<2 s_{\mathrm{X}} r_{\mathrm{m}}$ ), the influence of $f_{\mathrm{xx}}$, and consequently interveinal distance $s_{\mathrm{x}}$, remains actually very small. We believe that the absence of correlation is to be expected. Indeed, even the $\bar{\Delta}_{\text {lw }}$ values of Helliker \& Ehleringer (2000) exhibit a stronger difference between $\mathrm{C}_{3}$ and $\mathrm{C}_{4}$ groups alone $\left(1-\bar{\Delta}_{\mathrm{lw}} / \Delta_{\mathrm{C}}\right.$ about $30 \%$ lower for $\mathrm{C}_{4}$ plants) rather than within $\mathrm{C}_{3}$ or $\mathrm{C}_{4}$ difference. This is shown in Fig. 7 where we re-plotted the measured values of Helliker \& Ehleringer (2000), but grouped in terms of $\mathrm{C}_{3}$ and $\mathrm{C}_{4}$ plants to show that the apparent correlation with leaf dimension parameters was rather a difference between the two plant types. The distinction between the two groups is very clear in the data with the exception of one $\mathrm{C}_{4}$ plant (Muhlenbergia wrightii, marked by a larger circle on Fig. 7) that seems to behave more like a $C_{3}$ plant

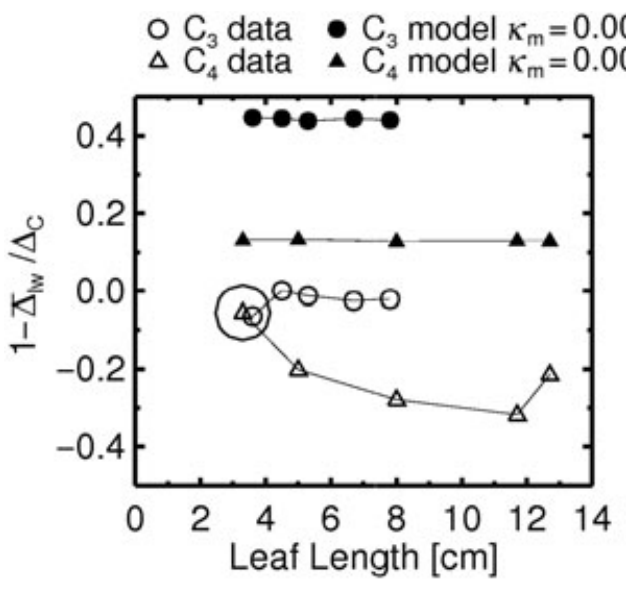

Figure 7. Bulk leaf water enrichment, expressed as deviation from the Craig-Gordon value, $1-\bar{\Delta}_{\mathrm{lw}} / \Delta_{\mathrm{C}}$, for a variety of plant species with different leaf length $l_{\max }$ and interveinal distance $s_{\mathrm{x}}$. Measured values (open symbols) reported by Helliker \& Ehleringer (2000) have been grouped between $\mathrm{C}_{3}$ and $\mathrm{C}_{4}$ plants to show that the apparent correlation with leaf dimension parameters was rather a difference between the two plant types. Modelled values (closed symbols) have been obtained using different mesophyll tortuosities between $\mathrm{C}_{3}$ and $\mathrm{C}_{4}$ plants. The larger circle shows the special behaviour of Muhlenbergia among the $\mathrm{C}_{4}$ group. 
(i.e. $1-\bar{\Delta}_{\text {lw }} / \Delta_{\mathrm{C}}$ is closer to zero like most $\mathrm{C}_{3}$ plants). The reason for this is not clear but Muhlenbergia is known to be quite a peculiar $\mathrm{C}_{4}$ plant in that it seems, unlike most $\mathrm{C}_{4}$ plants, to be relatively shade tolerant (Helliker, personal communication).

In the light of the sensitivity analysis conducted previously, we propose that this observed difference in steadystate $\bar{\Delta}_{\text {lw }}$ between $C_{3}$ and $C_{4}$ plants is due to differences in mesophyll tortuosity $\left(=1 / \kappa_{\mathrm{m}}\right)$. It is known that the leaves of most $\mathrm{C}_{4}$ plants differ from those of $\mathrm{C}_{3}$ plants in that they have two types of photosynthetic cells, the 'mesophyll' cells and the so-called Kranz or bundle-sheath cells (e.g. Hatch, Osmond \& Slatyer 1971; see also Voznesenskaya et al. 2002). It is also recognized that the bundle sheath cells are several order of magnitude less permeable to $\mathrm{CO}_{2}$ than other mesophyll cells (e.g. Furbank, Jenkins \& Hatch 1989). We believe that this specificity of $\mathrm{C}_{4}$ leaves (or more precisely leaves with Kranz anatomy) can also influence the diffusion of water isotopes. To test this hypothesis, we fitted values of $\kappa_{\mathrm{m}}$ so that the deviation between the $\mathrm{C}_{3}$ and $\mathrm{C}_{4}$ groups is the same as the one measured by Helliker \& Ehleringer (2000). The values are not unique but, if we keep a mesophyll tortuosity factor of 0.005 for $\mathrm{C}_{4}$ plants (the typical value in Table 1 for a corn leaf) then a smaller tortuosity factor of ca 0.001 for $\mathrm{C}_{3}$ plants is sufficient to increase $1-\bar{\Delta}_{\text {lw }} / \Delta_{\mathrm{C}}$ by about $30 \%$ compared to $\mathrm{C}_{4}$ plants and regardless of the values of $s_{\mathrm{x}}$ and $l_{\max }$ (Fig. 7). A higher tortuosity factor for $\mathrm{C}_{4}$ plants means a more linear water path, which does not necessarily go against a lower $\mathrm{CO}_{2}$ permeability of cell walls. In addition, for Muhlenbergia, we should expect a thinner mesophyll thickness $r_{\mathrm{m}}$ as in most shade-tolerant species. Reducing the mesophyll thickness by $50 \%$ for that species (and keeping $\kappa_{\mathrm{m}}=0.005$ ) changes the value of $1-\bar{\Delta}_{\mathrm{lw}} / \Delta_{\mathrm{C}}$ by the same order of magnitude as the one observed but in the other direction (i.e. -0.1 instead of +0.15 ). More work is surely needed to understand the link between $\kappa_{\mathrm{m}}, r_{\mathrm{m}}$ and leaf anatomy, and their influence on leaf water enrichment.

Even after fitting $\kappa_{\mathrm{m}}$ for the two plant types, there still remain discrepancies in Fig. 7 between our model and the measured values reported by Helliker \& Ehleringer (2000). This is because the measured values are nearly all above $\Delta_{\mathrm{C}}$, while our model always predicts values below $\Delta_{\mathrm{C}}$. From our current understanding, $\Delta_{\mathrm{C}}$ should be a maximum for mean flux-weighted leaf water enrichment at the sites of evaporation (Eqn 5; see also Appendix III in Farquhar \& Gan 2003) and consequently a maximum for bulk leaf water, so that $1-\bar{\Delta}_{\mathrm{lw}} / \Delta_{\mathrm{C}}$ should always be positive. Several reasons may explain the negative values reported by Helliker \& Ehleringer (2000). For example, increasing the value of $\varepsilon_{\mathrm{k}, 0}$ from 28.5 to $32 \%$ (Cappa et al. 2003) leads to higher CraigGordon values and therefore smaller deviations, which would then become positive for $\mathrm{C}_{3}$ plants but still slightly negative for $\mathrm{C}_{4}$ plants. It might also be that water entering the leaf was slightly enriched compared to tap water used to feed the whole plant. Indeed, in one experiment, Helliker \& Ehleringer (2000) reported a 3.1\% enrichment for water in the leaf petiole compared to tap water (i.e. $-12.5 \%$ instead of $-15.6 \%$ ). However, to get positive measured deviation for all the plants, this would require an enrichment in source water of up to $12 \%$ o (if we keep $\varepsilon_{\mathrm{k}, 0}=28.5 \%$ ), which is quite unrealistic. Non-uniform fractionation factors, induced by non-uniform stomatal conductance or leaf temperature, could also explain these differences in $1-\bar{\Delta}_{\mathrm{lw}} / \Delta_{\mathrm{C}}$. Overall, we believe that if all these effects were accounted for, $\bar{\Delta}_{\text {lw }}$ should never exceed $\Delta_{\mathrm{C}}$ (but surely reflects differences in mesophyll tortuosity between species).

\section{Model sensitivity to temporal variations of gas-exchange parameters}

So far the experimental data we have used to test our leaf water enrichment model have been obtained in the steady state only. Yet our model can also be tested against nonsteady-state measurements in order to see how the spatial patterns of $\Delta_{\text {lw }}$ vary in time due to changes in environmental conditions. For that we measured leaf water isotope enrichment patterns in a corn field at different times of the day, together with climate variables $\left(T_{\text {air }}, D_{\text {air }}\right)$, sap flow $(\bar{E})$, and isotope composition of water in the soil and water vapour in the air $\left(\Delta_{\mathrm{v}}\right)$. The experimental set-up is very similar to the one described in Bariac et al. (1994b).

A comparison of modelled and measured values of $\Delta_{\text {lw }}(l, t)$ at different times of the day is shown in Fig. 8. The model parameters used for these plots are as in Table 1, except for the gas-exchange parameters that were measured and the tortuosity factor that was fixed at 0.001 instead of 0.005 . The non-steady-state values, obtained with a uniform transpiration rate, are close to the experimental values except in the morning $(0900 \mathrm{~h})$ where the measurements exhibit a sharp and stronger enrichment around $l=0.8 l_{\max }$. This observed pattern is probably due to nonuniform transpiration rates and was reproduced by our model by increasing the transpiration rate in this region of the leaf (not shown). At other times of the day, the heterogeneity of the gas-exchange parameters is less obvious but could be invoked to tune the model to the experimental data. We also plotted the steady-state values of leaf water enrichment predicted by our model, that is, $\Delta_{\mathrm{lw}}(l, t \rightarrow \infty)$. A direct comparison of the modelled steady-state and nonsteady-state values shows that steady state is reached only in early afternoon (1300 and $1420 \mathrm{~h}$ ) and that non-steadystate effects are quite important at other times of the day. This result confirms the importance of non steady-state effects that had already been reported by previous studies on bulk leaf water enrichment (e.g. Bariac et al. 1991; Harwood et al. 1998; Cernusak et al. 2002; Farquhar \& Cernusak 2005).

Finally, an important issue is the extent to which these spatial patterns of $\Delta_{\mathrm{lw}}$ affect the bulk leaf water values and if simpler models, based on bulk leaf water budgets (e.g. Farquhar \& Cernusak 2005), can be used to describe leaf water enrichment variations of the whole leaf. To address this issue, we compared measured and modelled temporal variations of bulk leaf water $\bar{\Delta}_{\mathrm{lw}}$ using our non-steady-state, spatially explicit model and the non-steady-state bulk leaf 


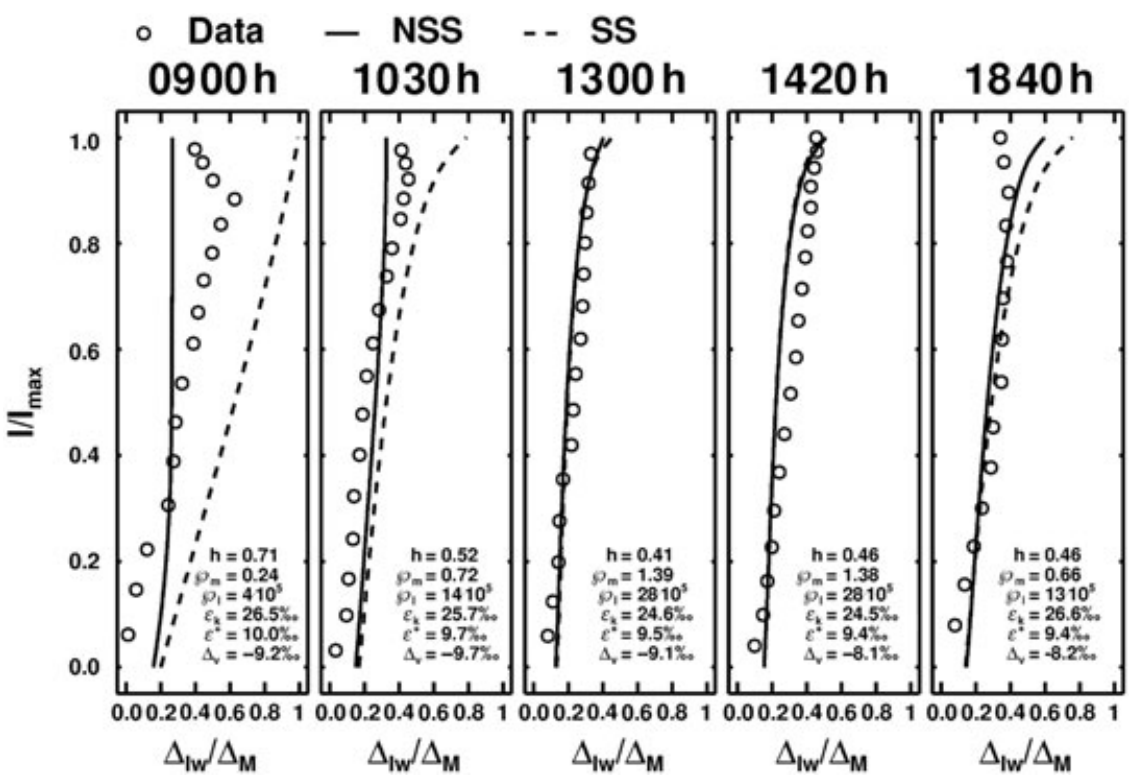

Figure 8. Leaf water isotope enrichment patterns $\left(\Delta_{\mathrm{lw}}\right)$ in a corn field at different times of the day, on 20 July 2000 at Grignon, France. Measurements (open circles) have been performed on different leaves taken from the top of the canopy and modelled values are computed either in the non-steady state (NSS, solid line) or in the steady state (SS, dotted line). water enrichment model of Farquhar \& Cernusak (2005). The results are shown in Fig. 9 and they demonstrate that the two non-steady-state models predict almost the same values of $\bar{\Delta}_{\text {lw }}$, and both are in good agreement with the measurements. However, some discrepancies between the two models are noticeable after dusk $(1900 \mathrm{~h})$ that we attributed to the sensitivity of the model of Farquhar \& Cernusak (2005) to the exact value of stomatal conductance before complete darkness. We plotted on the same figure the steady-state values computed with the Craig-Gordon equation (Eqn 5) and the spatially explicit model of Farquhar \& Gan (2003) to emphasize the fact that steady state is reached only between 1200 and $1800 \mathrm{~h}$ and that non-steadystate models are really needed at other times.

The good agreement between the two non-steady-state models is actually quite surprising. Indeed, if we assume again a uniform transpiration rate, our model equations can be easily integrated over the entire leaf and this leads to (see section on 'bulk non-steady-state isotope ratios' in Appendix II)

$V_{\mathrm{lw}} \frac{d \bar{\Delta}_{\mathrm{lw}}}{d t}=\frac{\bar{E}}{(1-h)} \cdot \frac{\bar{\Delta}_{\mathrm{e}}-\Delta_{\mathrm{C}}}{\alpha_{\mathrm{k}} \alpha^{+}}$,

where $V_{\mathrm{lw}}=C W /\left(2 s_{\mathrm{x}} l_{\max }\right)$ and $W=\left(a_{\mathrm{x}} l_{\max }+2 r_{\mathrm{m}} s_{\mathrm{x}} l_{\max }\right)$. Equation 30 is exactly eqn 5 in Farquhar \& Cernusak (2005) and forms the basis of their bulk leaf water enrichment model. However, to solve this equation they assumed that, at any time, $\bar{\Delta}_{\mathrm{lw}}$ and $\bar{\Delta}_{\mathrm{e}}$ stay proportional and that the coefficient of proportionality is equal to its steady-state value $f_{\mathrm{em}}$. In the bottom panel of Fig. 9, we plotted this ratio as predicted by the different models. We can see that our model predicts values of $\bar{\Delta}_{\mathrm{lw}} / \bar{\Delta}_{\mathrm{e}}$ that come very close to $f_{\mathrm{em}}$ about $1 \mathrm{~h}$ before a steady state is reached (around noon) and then remain close to $f_{\mathrm{em}}$ for the rest of the time series. This result shows that the assumed ratio of $\bar{\Delta}_{\mathrm{lw}} / \bar{\Delta}_{\mathrm{e}}$ in the bulk leaf model of Farquhar \& Cernusak (2005) seems valid during the day (late morning to early evening), even for long leaves with strong heterogeneous patterns in leaf water enrichment. This result is encouraging for large-scale studies.

\section{CONCLUSIONS AND PERSPECTIVES}

Overall, the two-dimensional model of isotopic leaf water enrichment presented in this study reproduced reasonably well all published steady-state measurements of leaf water enrichment along monocot leaves, except at the leaf tip. We show that longitudinal diffusion of liquid water in the mesophyll has very little effect on the isotopic gradient along the leaf, contrary to what has been proposed previously (Farquhar \& Gan 2003; Gan et al. 2003). We also believe that transport in the vapour phase, not accounted for in our model, should not have a much stronger effect. The observed reduction in the isotopic gradient at the leaf tip seems to result rather from the combination of several (non-diffusive) effects such as non-uniform transpiration rate, tapering of the leaf blade or the leaf veins and changing leaf water volume. However, most of these effects are difficult to account for, given the simplicity of the model leaf geometry. Currently, this model leaf geometry is adapted to amphistomatous monocot species only, such as most crops (e.g. corn) and wild grasses, but it can be easily modified to represent fully cylindrical coniferous needles. Because needles usually have one single vein, the tapering of the leaf is quite limited. It would seem therefore interesting to test the model against $\Delta_{\mathrm{lw}}$ data collected on such long needles and see how it behaves at distances above $0.8-0.9 l_{\max }$.

Contrary to what has been proposed before (Helliker \& Ehleringer 2000), our model suggests that the isotopic enrichment remains quite insensitive to leaf length $l_{\max }$ and interveinal distance $s_{\mathrm{x}}$ and also to xylem tortuosity $\left(=1 / \kappa_{\mathrm{x}}\right)$. 


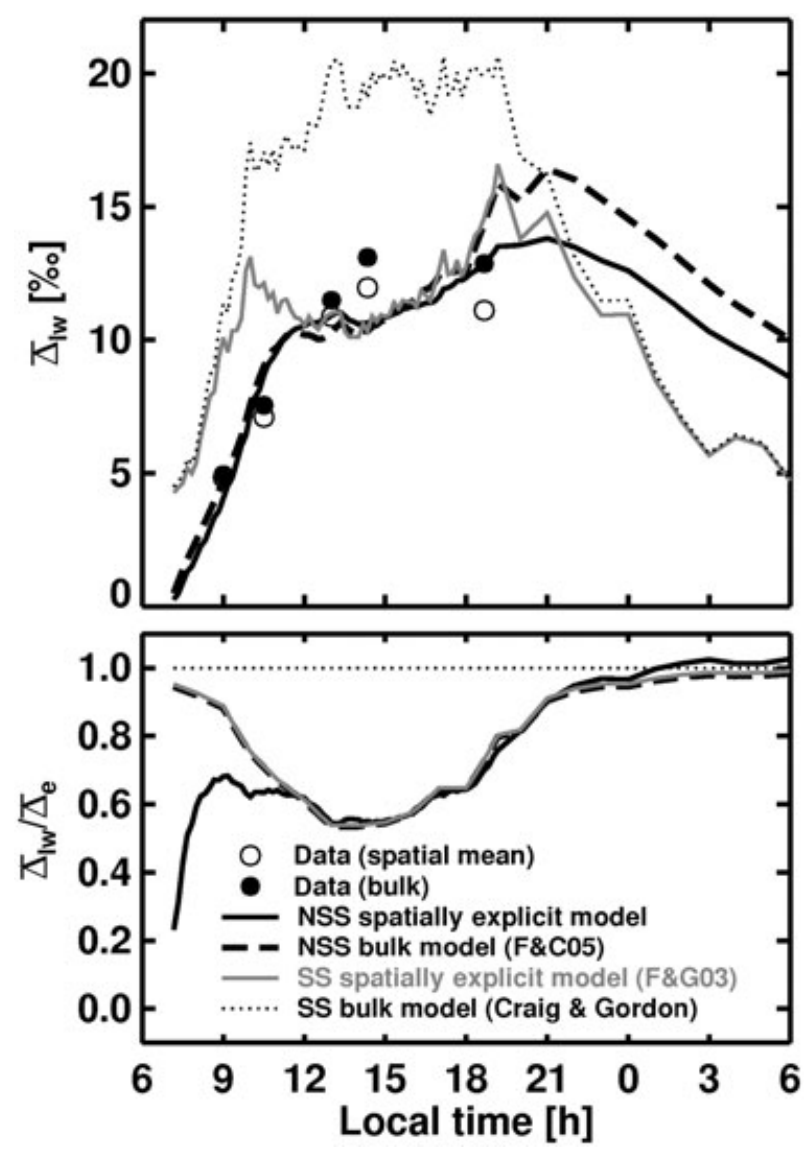

Figure 9. Diurnal cycle of bulk leaf water isotope enrichment $\left(\bar{\Delta}_{\mathrm{lw}}\right)$ and its ratio to bulk leaf water enrichment at the evaporative sites $\left(\bar{\Delta}_{\mathrm{e}}\right)$ in a corn field at different times of the day, on 20 July 2000 at Grignon, France. Measurements have been performed on entire leaves taken from the top of the canopy (filled circles) or deduced from spatially explicit measurements shown in Fig. 8 (open circles). Modelled values are computed with the non-steady-state (NSS), spatially explicit model (thick solid line), the NSS, bulk leaf water enrichment of Farquhar \& Cernusak (2005) (F\&C05, thick dashed line) or the steady-state (SS), spatially explicit model of Farquhar \& Gan (2003) (F\&G03, thin solid grey line). Craig-Gordon values are also shown (thin dotted line).

Indeed, spatial patterns of $\Delta_{\mathrm{lw}}$ along monocot leaves are mostly controlled by mesophyll tortuosity $\left(=1 / \kappa_{\mathrm{m}}\right)$. It is also the only parameter that can explain observed differences of bulk leaf water enrichment between $\mathrm{C}_{3}$ and $\mathrm{C}_{4}$ plants, with a lower tortuosity factor $\kappa_{\mathrm{m}}$ for $\mathrm{C}_{3}$ plants. In fact, the so-called Kranz anatomy, which is specific to most $\mathrm{C}_{4}$ species, is likely to influence mesophyll tortuosity.

However, mesophyll tortuosity does not seem to be species-specific only as different values of $\kappa_{\mathrm{m}}$ were required for the same species with different growth and environmental conditions. In the two experiments conducted on fieldgrown plants, $\kappa_{\mathrm{m}}$ seemed consistently to have smaller values. This might be due to changes in leaf water volume or in the water path (Barbour \& Farquhar 2003). To better understand the meaning of the parameter $\kappa_{\mathrm{m}}$, it would be interesting to test our model against data collected in the dark, when transpiration is reduced, because tracer diffusion would then become the main process to explain variations of $\Delta_{\text {lw }}$ in space and time.

Our results also suggest that non-steady-state effects on the spatial patterns of $\Delta_{\mathrm{lw}}$ are quite important in the field, except at midday when leaf transpiration is at maximum. However, these non-steady-state effects do not seem to greatly affect the ratio $\bar{\Delta}_{\text {lw }} / \bar{\Delta}_{\mathrm{e}}$ that remains close to its steady-state value. This is very useful for upscaling the results to the bulk leaf level, as was done in Farquhar \& Cernusak (2005). However, these non-steady-state effects on the ratio $\bar{\Delta}_{\mathrm{lw}} / \bar{\Delta}_{\mathrm{e}}$ should be studied more thoroughly to better characterize the situations when this ratio deviates from its steady-state value. Moreover, it would be interesting to couple our leaf water enrichment model to a leaf growth and cellulose synthesis model to know to what extent leaf cellulose imprints leaf water isotope signals during leaf development and if this cellulose signal can be interpreted in terms of growth humidity levels or other climatic variables.

\section{ACKNOWLEDGMENTS}

We would like to thank K.S. Gan, B.R. Helliker and D. Yakir for providing us their data sets. We also thank G.D. Farquhar, B.R. Helliker and two anonymous reviewers for valuable comments on an earlier version of this manuscript. M. Cuntz is supported by a European Union Marie-Curie Fellowship (contract number MOIF-CT-2004-008087).

\section{REFERENCES}

Affek H.P., Krisch M.J. \& Yakir D. (2006) Effects of intraleaf variations in carbonic anhydrase activity and gas exchange on leaf $\mathrm{C}^{18} \mathrm{OO}$ isoflux in Zea mays. New Phytologist 169, 321-329.

Barbour M.M. \& Farquhar G.D. (2003) Do pathways of water movement and leaf anatomical dimensions allow development of gradients in $\mathrm{H}_{2}{ }^{18} \mathrm{O}$ between veins and the sites of evaporation within the leaves? Plant, Cell \& Environment 27, 1-15.

Barbour M.M., Schurr U., Henry B.K., Wong S.C. \& Farquhar G.D. (2000) Variation in the oxygen isotope ratio of phloem sap sucrose from castor bean. Evidence in support of the Péclet effect. Plant Physiology 123, 671-679.

Bariac T. (1987) Evolutions de l'oxygène-18 et du deuterium de l'eau dans le continuum sol-plante-atmosphère. Bulletin $d u$ G.F.H.N. 21, 11-33.

Bariac T., Jusserand C. \& Mariotti A. (1990) Evolution spatiotemporelle de la composition isotopique de l'eau dans le continuum sol-plante-atmosphère. Geochimica et Cosmochimica Acta 54, 413-424.

Bariac T., Deleens E., Gerbaud A., Andre M. \& Mariotti A. (1991) La composition isotopique $\left({ }^{18} \mathrm{O},{ }^{2} \mathrm{H}\right)$ de la vapeur d'eau transpirée: etude en conditions asservies. Geochimica et Cosmochimica Acta 55, 3391-3402.

Bariac T., Gonzalez-Dunia J., Tardieu F., Tessier D. \& Mariotti A. (1994a) Variabilité spatiale de la composition isotopique de l'eau $\left({ }^{18} \mathrm{O},{ }^{2} \mathrm{H}\right)$ au sein des organes des plantes aériennes: 1 . Approche en conditions controlées: spatial variation of the isotopic composition of water $\left({ }^{18} \mathrm{O},{ }^{2} \mathrm{H}\right)$ in organs of aerophytic plants: 1 . Assessment under laboratory conditions. Chemical Geology 115, 307-315. 
Bariac T., Gonzalez-Dunia J., Katerji N., Bethenod O., Bertolini J.M. \& Mariotti A. (1994b) Variabilité spatio-temporelle de la composition isotopique de l'eau $\left({ }^{18} \mathrm{O},{ }^{2} \mathrm{H}\right)$ dans le continuum solplante-atmosphere 2. Approche en conditions naturelles: spatial variation of the isotopic composition of water $\left({ }^{18} \mathrm{O},{ }^{2} \mathrm{H}\right)$ in the soil-plant-atmosphere system. 2. Assessment under field conditions. Chemical Geology 115, 317-333.

Barnes B., Farquhar G.D. \& Gan K. (2004) Modelling the isotope enrichment of leaf water. Journal of Mathematical Biology 48, 672-702.

Buckley T.N. \& Mott K.A. (2000) Stomatal responses to non-local changes in PFD: evidence for long-distance hydraulic interactions. Plant, Cell \& Environment 23, 301-309.

Cappa C.D., Hendricks M.B., DePaolo D.J. \& Cohen R.C. (2003) Isotopic fractionation of water during evaporation. Journal of Geophysical Research 108 (D16), 4525. doi: 10.1029/ 2003JD003597

Cernusak L.A., Pate J.S. \& Farquhar G.D. (2002) Diurnal variation in the stable isotope composition of water and dry matter in fruiting Lupinus angustifolius under field conditions. Plant, Cell \& Environment 25, 893-907.

Dongmann G., Nürnberg H.W., Förstel H. \& Wagener K. (1974) On the enrichment of $\mathrm{H}_{2}{ }^{18} \mathrm{O}$ in the leaves of transpiring plants. Radiation and Environmental Biophysics 11, 41-52.

Easteal A.J., Edge A.V.J. \& Woolf, L.A. (1984) Isotope effects in water. Tracer diffusion coefficients for $\mathrm{H}_{2}{ }^{18} \mathrm{O}$ in ordinary water. Journal of Physical Chemistry 88, 6060-6063.

Farquhar G.D. \& Cernusak L.A. (2005) On the isotopic composition of leaf water in the non-steady state. Functional Plant Biology 32, 293-303.

Farquhar G.D. \& Gan K.S. (2003) On the progressive enrichment of the oxygen isotopic composition of water along a leaf. Plant, Cell \& Environment 26, 1579-1597.

Farquhar G.D. \& Lloyd J. (1993) Carbon and oxygen isotope effects in the exchange of carbon dioxide between terrestrial plants and the atmosphere. In Stable Isotopes and Plant Carbon-Water Relations (eds J.R. Ehleringer, A.E. Hall \& G.D. Farquhar), pp. 47-70. Academic Press, San Diego, CA, USA.

Farquhar G.D., Hubick K.T., Condon A.G. \& Richards R.A. (1989) Carbon isotope fractionation and plant water-use efficiency. In Stable Isotopes in Ecological Research (eds P.W. Rundel, J.R. Ehleringer \& K.A. Nagy), pp. 21-40. Springer-Verlag, New York, NY, USA.

Flanagan L.B. (1993) Environmental and biological influences on the stable oxygen and hydrogen isotopic composition of leaf water. In Stable Isotopes and Plant Carbon-Water Relations (eds J.R. Ehleringer, A.E. Hall \& G.D. Farquhar), pp. 71-90. Academic Press, San Diego, CA, USA.

Flanagan L.B., Comstock J.P. \& Ehleringer J.R. (1991) Comparison of modeled and observed environmental influences on the stable oxygen and hydrogen isotope composition of leaf water in Phasoleus vulgaris. Plant Physiology 96, 588-596.

Fontes J.-C. \& Gonfiantini R. (1967) Comportement isotopique au cours de l'evaporation de deux bassins sahariens. Earth and Planetary Science Letters 3, 258-266.

Furbank R.T., Jenkins C.L.D. \& Hatch M.D. (1989) $\mathrm{CO}_{2}$ concentrating mechanism of $\mathrm{C}_{4}$ photosynthesis: permeability of isolated bundle sheath cells to inorganic carbon. Plant Physiology 91, 1364-1371.

Gan K.S., Wong S.C., Yong J.W.H. \& Farquhar G.D. (2002) ${ }^{18} \mathrm{O}$ spatial patterns of vein xylem water, leaf water and dry matter in cotton leaves. Plant Physiology 130, 1008-1021.

Gan K.S., Wong S.C., Yong J.W.H. \& Farquhar G.D. (2003) Evaluation of models of leaf water ${ }^{18} \mathrm{O}$ enrichment using measurements of spatial patterns of vein xylem, leaf water and dry matter in maize leaves. Plant, Cell \& Environment 26, 1479-1495.
Gat J.R. \& Bowser C. (1991) The heavy isotope enrichment of water in coupled evaporative systems. In Stable Isotope Geochemistry: A Tribute to Samuel Epstein (eds H.P. Taylor, J.R. O'Neil, \& I.R. Kaplan), pp. 159-168. The Geochemistry Society, St Louis, MO, USA.

Harwood K.G., Gillon J.S., Griffiths H. \& Broadmeadow M.S.J. (1998) Diurnal variation of $\Delta{ }^{13} \mathrm{CO}_{2}, \Delta \mathrm{C}^{18} \mathrm{O}^{16} \mathrm{O}$ and evaporative site enrichment of $\Delta \mathrm{H}_{2}{ }^{18} \mathrm{O}$ in Piper aduncum under field conditions in Trinidad. Plant, Cell \& Environment 21, 269-283.

Hatch M.D., Osmond C.B. \& Slatyer R.O. (1971) Photosynthesis and Photorespiration. Wiley InterScience, New York, NY, USA.

Helliker B.R. \& Ehleringer J.R. (2000) Establishing a grassland signature in veins: ${ }^{18} \mathrm{O}$ in the leaf water of $\mathrm{C} 3$ and $\mathrm{C} 4$ grasses. Proceedings of the National Academy of Science of USA 97, 7894-7898.

Helliker B.R. \& Ehleringer J.R. (2002) Grass blades as tree rings: environmentally induced changes in the oxygen isotope ratio of cellulose along the length of grass blades. New Phytologist 155, 417-424.

Horita J. \& Wesolowski D.J. (1994) Liquid-vapor fractionation of oxygen and hydrogen isotopes of water from the freezing to the critical temperature. Geochimica et Cosmochimica Acta 58, 3425-3437.

Luo Y.H. \& Sternberg L. (1992) Spatial D/H heterogeneity of leaf water. Plant Physiology 99, 348-350.

Majoube M. (1971) Fractionnement en oxygène 18 et en deutérium entre l'eau et sa vapeur. Journal de Chimie Physique 68, 14231436.

Mathieu R. \& Bariac T. (1996) A numerical model for the simulation of stable isotope profiles in drying soils. Journal of Geophysical Research 101, 12685-12696.

Meinzer F.C. \& Salendra N.Z. (1997) Spatial patterns of carbon isotope discrimination and allocation of photosynthetic activity in sugarcane leaves. Australian Journal of Plant Physiology 24, 767-775.

Merlivat L. (1978) Molecular diffusivities of $\mathrm{H}_{2}{ }^{16} \mathrm{O}, \mathrm{HD}^{16} \mathrm{O}$ and $\mathrm{H}_{2}{ }^{18} \mathrm{O}$ in gases. Journal of Chemical Physics 69, 2864-2871.

Mott K.A. \& Buckley T.N. (1998) Stomatal heterogeneity. Journal of Experimental Botany 49, 407-417.

Patankar S.V. (1980) Numerical Heat Transfer and Fluid Flow. Hemisphere Publishing Corporation, New York, NY, USA.

Reinecke S.A. \& Sleep B.E. (2002) Knudsen diffusion, gas permeability, and water content in an unconsolidated porous medium. Water Resources Research 38, 1280, doi:1210.1029/ 2002WR001278.

Roden J.S. \& Ehleringer J.R. (1999) Observations of hydrogen and oxygen isotopes in leaf water confirm the Craig-Gordon model under wide-ranging environmental conditions. Plant Physiology 120, 1165-1173.

Schäffner A.R. (1998) Aquaporin function, structure and expression: are there more surprises in water relations? Planta 204, 131-139.

Voznesenskaya E.V., Franceschi V.R., Kiirats O., Artyusheva E.G., Freitag H. \& Edwards G.E. (2002) Proof of C4 photosynthesis without Kranz anatomy in Bienertia cycloptera (Chenopodiaceae). The Plant Journal 31, 649-662.

Wang X.F. \& Yakir D. (1995) Temporal and spatial variations in the oxygen-18 content of leaf water in different plant species. Plant, Cell \& Environment 18, 1377-1385.

Wright W.E. \& Leavitt S.W. (2006) Needle cell elongation and maturation timing derived from pine needle cellulose $\delta^{18} \mathrm{O}$. Plant, Cell \& Environment 29, 1-14.

Yakir D. (1992) Water compartmentation in plant tissue: isotopic evidence. In Water and Life (eds G.N. Somero, C.B. Osmond \& L. Bolis), pp. 205-222. Springer-Verlag, Berlin, Germany.

Yakir D. (1998) Oxygen-18 of leaf water: a crossroad for 
plant-associated isotopic signals. In Stable Isotopes (ed. H. Griffiths), pp. 147-168. BIOS Scientific Publishers Ltd., Oxford, UK.

Yakir D., DeNiro M. \& Rundel P.W. (1989) Isotopic inhomogeneity of leaf water: evidence and implications for the use of isotope signals transduced by plants. Geochimica and Cosmochimica Acta 53, 2769-2773.

Zhu F. \& Schulten K. (2003) Water and proton conduction through carbon nanotubes as models for biological channels. Biophysical Journal 85, 236-244.

Received 20 July 2006; received in revised form 27 October 2006; accepted for publication 17 November 2006

\section{APPENDIX I}

\section{List of symbols}

$a_{\mathrm{x}} \quad$ Vein cross-sectional area $\left(\mathrm{m}^{2}\right)$

$C \quad$ Molar concentration of liquid water $\left[\mathrm{mol}\left(\mathrm{H}_{2} \mathrm{O}\right) \mathrm{m}^{-3}\right]$

$D$ Diffusivity of isotopologues in 'normal' water $\left(\mathrm{m}^{2} \mathrm{~s}^{-1}\right)$

$D_{\text {air }} \quad$ Vapour pressure deficit $(\mathrm{Pa})$

$D_{\operatorname{lm}} \quad$ Effective diffusivity in mesophyll in longitudinal direction, if different from $D_{\mathrm{m}}\left(\mathrm{m}^{2} \mathrm{~s}^{-1}\right)$

$D_{\mathrm{m}} \quad$ Effective diffusivity in mesophyll $\left(\mathrm{m}^{2} \mathrm{~s}^{-1}\right)$

$D_{\mathrm{x}} \quad$ Effective diffusivity in xylem in radial direction $\left(\mathrm{m}^{2} \mathrm{~s}^{-1}\right)$

$E \quad$ Transpiration rate $\left[\mathrm{mol}\left(\mathrm{H}_{2} \mathrm{O}\right) \mathrm{m}^{2} \mathrm{~s}^{-1}\right]$

$\bar{E} \quad$ Longitudinal mean transpiration rate $\left[\mathrm{mol}\left(\mathrm{H}_{2} \mathrm{O}\right) \mathrm{m}^{2} \mathrm{~s}^{-1}\right]$

$f_{\mathrm{em}} \quad$ Factor relating bulk mesophyll water enrichment with enrichment at the evaporating site

$f_{\mathrm{E}} \quad$ Normalized function for non-uniform transpiration rate in longitudinal direction

$f_{\min } \quad$ Minimum of normalized functions for non-uniform gas-exchange parameters if described with Gaussian profile

$f_{\mathrm{g}} \quad$ Normalized function for non-uniform stomatal conductance in longitudinal direction

$f_{\mathrm{T}} \quad$ Normalized function for non-uniform leaf temperature in longitudinal direction

$f_{\mathrm{xx}} \quad$ Factor relating bulk xylem water enrichment with enrichment at xylem-mesophyll interface

Boundary-layer conductance [ $\mathrm{mol}($ air $) \mathrm{m}^{-2} \mathrm{~s}^{-1}$ ]

Stomatal conductance $\left[\mathrm{mol}(\mathrm{air}) \mathrm{m}^{-2} \mathrm{~s}^{-1}\right.$ ]

Longitudinal mean stomatal conductance [mol(air) $\mathrm{m}^{-2} \mathrm{~s}^{-1}$ ]

Total conductance $\left[\mathrm{mol}(\right.$ air $) \mathrm{m}^{-2} \mathrm{~s}^{-1}$ ]

Relative humidity corrected to leaf temperature

Relative humidity corrected to leaf temperature and fractionations

Cumulative transpiration rate from $l$ to $l_{\max }\left[\operatorname{mol}\left(\mathrm{H}_{2} \mathrm{O}\right)\right.$ $\left.\mathrm{m}^{-1} \mathrm{~s}^{-1}\right]$

$I_{\max } \quad$ Cumulative transpiration rate from petiole to $l_{\max }$ $\left[\mathrm{mol}\left(\mathrm{H}_{2} \mathrm{O}\right) \mathrm{m}^{-1} \mathrm{~s}^{-1}\right]$

$k \quad$ Exponent in desert river model including radial diffusion

$l \quad$ Longitudinal coordinate $(\mathrm{m})$

$l_{\max } \quad$ Leaf length $(\mathrm{m})$

$l_{\text {opt }} \quad$ Length at which the mean of normalized functions for non-uniform gas exchange parameters occurs if described with Gaussian profile $(\mathrm{m})$

$L_{\mathrm{m}} \quad$ Effective length in mesophyll (m)

$P_{\text {air }} \quad$ Air pressure $(\mathrm{Pa})$

$\wp_{1} \quad$ Longitudinal Péclet number in xylem

$\wp_{\mathrm{m}} \quad$ Radial Péclet number in mesophyll

$\wp_{\mathrm{x}} \quad$ Radial Péclet number in xylem

$r \quad$ Radial coordinate $(\mathrm{m})$

$r_{\mathrm{e}} \quad$ Radial coordinate evaporation sites $(\mathrm{m})$

$r_{\mathrm{m}} \quad$ Mesophyll half-thickness (m) $r_{\max } \quad$ Maximum value of $r(\mathrm{~m})$

$r_{\mathrm{x}} \quad$ Xylem radius $(\mathrm{m})$

$r_{\mathrm{x}}^{-} \quad$ Radial coordinate $r$ at xylem radius, in xylem (m)

$r_{\mathrm{x}}^{+} \quad$ Radial coordinate $r$ at xylem radius, in mesophyll (m)

$R \quad$ Isotope ratio

$\bar{R} \quad$ Longitudinal mean isotope ratio

$R_{\mathrm{C}} \quad$ Craig-Gordon isotope ratio

$R_{\mathrm{e}} \quad$ Isotope ratio at evaporative site

$\bar{R}_{\mathrm{e}} \quad$ Longitudinal mean isotope ratio at evaporative site

$R_{\mathrm{E}} \quad$ Isotope ratio of transpiration

$\bar{R}_{\mathrm{E}} \quad$ Longitudinal mean isotope ratio of transpiration

$R_{\mathrm{lw}} \quad$ Radial mean bulk isotope ratio (xylem plus mesophyll)

$\bar{R}_{\mathrm{lw}} \quad$ Longitudinal and radial mean bulk isotope ratio (xylem plus mesophyll)

$R_{\mathrm{m}} \quad$ Radial mean mesophyll isotope ratio

$\bar{R}_{\mathrm{m}} \quad$ Longitudinal and radial mean mesophyll isotope ratio

$R_{\mathrm{M}} \quad$ Maximum possible leaf water enrichment isotope ratio

$R_{\mathrm{S}} \quad$ Isotope ratio of source water from the petiole

$R_{\mathrm{v}} \quad$ Isotope ratio of ambient water vapour

$R_{\mathrm{x}} \quad$ Radial mean xylem isotope ratio

$\bar{R}_{\mathrm{X}} \quad$ Longitudinal and radial mean xylem isotope ratio

$s_{\mathrm{X}} \quad$ Distance between lateral veins (m)

$t \quad$ Time coordinate (s)

$T_{\text {air }} \quad$ Ambient air temperature (K)

$T_{\text {leaf }} \quad$ Leaf temperature (K)

$\bar{T}_{\text {leaf }} \quad$ Longitudinal mean leaf temperature $(\mathrm{K})$

$\boldsymbol{v} \quad$ Advection velocity vector $\left(\mathrm{m} \mathrm{s}^{-1}\right)$

$v_{1} \quad$ Advection velocity in longitudinal direction $\left(\mathrm{m} \mathrm{s}^{-1}\right)$

$v_{\mathrm{m}} \quad$ Radial advection velocity in mesophyll $\left(\mathrm{m} \mathrm{s}^{-1}\right)$

$v_{\mathrm{r}} \quad$ Advection velocity in radial direction $\left(\mathrm{m} \mathrm{s}^{-1}\right)$

$\bar{v}_{\mathrm{r}} \quad$ Longitudinal mean advection velocity in radial direction $\left(\mathrm{m} \mathrm{s}^{-1}\right)$

$v_{\mathrm{x}} \quad$ Radial mean longitudinal advection velocity in xylem $\left(\mathrm{m} \mathrm{s}^{-1}\right)$

$v_{\mathrm{x} 0} \quad$ Longitudinal advection velocity in xylem at leaf base $\left(\mathrm{m} \mathrm{s}^{-1}\right)$

$w_{\mathrm{a}} \quad$ Ambient humidity $\left[\mathrm{mol}\left(\mathrm{H}_{2} \mathrm{O}\right) \operatorname{mol}(\text { air })^{-1}\right]$

$w_{\mathrm{i}} \quad$ Humidity in the stomatal cavity $\left[\mathrm{mol}\left(\mathrm{H}_{2} \mathrm{O}\right) \operatorname{mol}(\text { air })^{-1}\right]$

$W \quad$ Leaf water volume $\left(\mathrm{m}^{3}\right)$

$W_{\mathrm{lw}} \quad$ Leaf water volume per leaf area $\left[\mathrm{mol}\left(\mathrm{H}_{2} \mathrm{O}\right) \mathrm{m}^{-2}\right]$

$\alpha^{+} \quad$ Equilibrium water-vapour fractionation factor

$\alpha_{\mathrm{k}} \quad$ Kinetic fractionation factor through stomata and boundary layer

$\delta_{\mathrm{s}} \quad$ Isotope ratio of source water from the petiole relative to VSMOW

$\delta_{\mathrm{V}} \quad$ Isotope ratio of ambient water vapour relative to VSMOW

$\delta l \quad$ Longitudinal dimension of elemental cylinder $(\mathrm{m})$

$\delta r \quad$ Radial dimension of elemental cylinder (m)

$\delta t \quad$ Infinitesimally small time step (s)

$\Delta \quad$ Isotope ratio relative to source water

$\bar{\Delta} \quad$ Longitudinal mean isotope ratio relative to source water

$\Delta_{\mathrm{C}} \quad$ Craig-Gordon isotope ratio relative to source water

$\Delta_{\mathrm{e}} \quad$ Isotope ratio at evaporative site relative to source water

$\bar{\Delta}_{\mathrm{e}} \quad$ Longitudinal mean isotope ratio at evaporative site relative to source water

$\Delta_{\mathrm{E}} \quad$ Isotope ratio of transpiration relative to source water

$\bar{\Delta}_{\mathrm{E}} \quad$ Longitudinal mean isotope ratio of transpiration relative to source water

$\Delta_{\text {lw }} \quad$ Radial mean bulk isotope ratio relative to source water (xylem plus mesophyll)

$\bar{\Delta}_{\mathrm{lw}} \quad$ Longitudinal and radial mean bulk isotope ratio relative to source water (xylem plus mesophyll)

$\Delta_{\mathrm{m}} \quad$ Radial mean mesophyll isotope ratio relative to source water 
$\bar{\Delta}_{\mathrm{m}} \quad$ Longitudinal and radial mean mesophyll isotope ratio relative to source water

$\Delta_{\mathrm{v}} \quad$ Isotope ratio of ambient water vapour relative to source water

$\Delta_{\mathrm{x}} \quad$ Radial mean xylem isotope ratio relative to source water

$\bar{\Delta}_{\mathrm{x}} \quad$ Longitudinal and radial mean xylem isotope ratio relative to source water

Equilibrium water-vapour fractionation

$\varepsilon_{\mathrm{k}} \quad$ Kinetic fractionation through stomata and boundary layer

$\varepsilon_{\mathrm{k} 0} \quad$ Kinetic fractionation through stomata

$\phi_{\mathrm{x}} \quad$ Volume fraction of leaf occupied by veins

$\kappa_{\mathrm{m}} \quad$ Tortuosity factor in mesophyll

$\kappa_{\mathrm{x}} \quad$ Radial tortuosity factor in xylem

$\sigma_{1} \quad \mathrm{SD}$ of normalized functions for non-uniform gas-exchange parameters if described with Gaussian profile (m)

\section{APPENDIX II}

\section{Model description}

\section{Water velocity field (Eqn 15 and Eqn 19)}

The continuity equation (Eqn 13) in the cylindrical veins can be written as

$\frac{\partial v_{1}}{\partial l}+\frac{1}{r} \frac{\partial r v_{\mathrm{r}}}{\partial r}=0$

If we assume that $v_{1}(r, l, t)$ is independent of $r$, then $v_{1}(r, l, t)=v_{\mathrm{x}}(l, t)$ and

$$
\left.\frac{\partial r v_{\mathrm{r}}}{\partial r}\right|_{r, l, t}=-\left.r \frac{\partial v_{\mathrm{x}}}{\partial l}\right|_{l, t}
$$

The integration of this equation (from 0 to $r$ ) leads to

$r v_{\mathrm{r}}(r, l, t)=-\left.\frac{r^{2}}{2} \frac{\partial v_{\mathrm{x}}}{\partial l}\right|_{l, t}$,

which leads to Eqn 15 (by setting $r=r_{\mathrm{x}}$ ) and Eqn 19.

\section{Water isotope ratio in the xylem (Eqn 20)}

The continuity equation for the isotope ratio in the xylem can be derived in the same manner as for total liquid water. However, we must now account for back diffusion fluxes in the radial and longitudinal directions. For an elemental cylinder of radius $r \leq r_{\mathrm{x}}$, thickness $\delta r$ and length $\delta l$ in the leaf xylem, the quantity of water isotopes in this element is $R C \times 2 \pi r \delta r \delta l(\mathrm{~mol})$, where $2 \pi r \delta r \delta l\left(\mathrm{~m}^{3}\right)$ is the volume of the elemental cylinder. This quantity changes due to water isotope fluxes in and out of the element. For example, the quantity of water isotopes that enters this element at $l$ during time $\delta t$ is $R C \times v_{1}(r, l, t) \times 2 \pi r \delta r \delta t$ (recalling that $2 \pi r \delta r$ is the area crossed by the longitudinal advection flux) and the quantity of water isotopes that back-diffuses at $l$ during time $\delta t$ is $D \cdot \partial R C / \partial l_{\mathrm{r}, 1, \mathrm{t}} \times 2 \pi r \delta r \delta t$. To write the water isotope budget of the elemental cylinder, we must account for all the different fluxes at the boundaries of this element. This leads to

$$
\begin{aligned}
\delta R(r, l, t) \cdot 2 \pi r \delta r \delta l= & +v_{\mathrm{l}} R(r, l, t) \cdot 2 \pi r \delta r \delta t \\
& -D \partial R /\left.\partial l\right|_{r, l, t} \cdot 2 \pi r \delta r \delta t \\
& -v_{\mathrm{l}} R(r, l+\delta l, t) \cdot 2 \pi r \delta r \delta t \\
& -D \partial R /\left.\partial l\right|_{r, l+\delta l, t} \cdot 2 \pi r \delta r \delta t \\
& +v_{\mathrm{r}} R(r, l, t) \cdot 2 \pi r \delta l \delta t \\
& -\kappa_{\mathrm{x}} D \partial R /\left.\partial r\right|_{r, l, t} \cdot 2 \pi r \delta l \delta t \\
& -v_{\mathrm{r}} R(r+\delta r, l, t) \cdot 2 \pi[r+\delta r] \delta l \delta t \\
& +\kappa_{\mathrm{x}} D \partial R /\left.\partial r\right|_{r+\delta r, l, t} \cdot 2 \pi[r+\delta r] \delta l \delta t
\end{aligned}
$$

Dividing this equation by $\delta t$ and making a first-order Taylor expansion of the velocities and the isotope ratios gives

$$
\begin{aligned}
\frac{\partial R}{\partial t} \cdot 2 \pi r \delta r \delta l= & +v_{1} R \cdot 2 \pi r \delta r \\
& -D \partial R / \partial l \cdot 2 \pi r \delta r \\
& -\left\{v_{1} R+\partial v_{1} R / \partial l \cdot \delta l\right\} \cdot 2 \pi r \delta r \\
& +D\left\{\partial R / \partial l+\partial^{2} R / \partial l^{2} \cdot \delta l\right\} \cdot 2 \pi r \delta r \\
& +v_{\mathrm{r}} R \cdot 2 \pi r \delta l \\
& -\kappa_{\mathrm{x}} D \partial R / \partial r \cdot 2 \pi r \delta l \\
& -\left\{v_{\mathrm{r}} R+\partial v_{\mathrm{r}} R / \partial r \cdot \delta r\right\} \cdot 2 \pi[r+\delta r] \delta l \\
& +\kappa_{\mathrm{x}} D\left\{\partial R / \partial r+\partial^{2} R / \partial r^{2} \cdot \delta r\right\} \cdot 2 \pi[r+\delta r] \delta l
\end{aligned}
$$

Finally if we neglect the second-order terms and divide by the volume $2 \pi r \delta r \delta l$, we get

$$
\frac{\partial R}{\partial t}=-\frac{\partial v_{1} R}{\partial l}-\frac{v_{\mathrm{r}} R}{r}-\frac{\partial v_{\mathrm{r}} R}{\partial r}+D\left\{\frac{\partial^{2} R}{\partial l^{2}}+\frac{\kappa_{\mathrm{x}}}{r} \frac{\partial R}{\partial r}+\frac{\partial^{2} R}{\partial r^{2}}\right\},
$$

which can be easily rearranged to obtain Eqn 20 in the main text.

\section{Water isotope ratio in the mesophyll (Eqn 22)}

The continuity equation for water isotopes in the mesophyll is derived in the same manner as in the xylem but for a planar elemental element and no longitudinal advection. This leads to

$$
\frac{\partial R C}{\partial t}=-\frac{\partial v_{\mathrm{r}} R C}{\partial r}+D_{\mathrm{m}}\left\{\frac{\partial}{\partial r}\left(\frac{\partial R C}{\partial r}\right)+\frac{\partial}{\partial l}\left(\frac{\partial R C}{\partial l}\right)\right\} .
$$

Dividing by $C$ and rearranging leads exactly to Eqn 22 in the main text.

\section{Bulk steady-state isotope ratios assuming $f_{\mathrm{E}}(I)=1$ (Eqn 27 and seq.)}

\section{Bulk isotope ratio in the xylem}

The bulk isotope ratio in the leaf longitudinal xylem is computed as follows:

$$
\bar{R}_{\mathrm{x}}(t)=\frac{1}{l_{\max }} \int_{0}^{l_{\max }} d l \cdot\left\{\frac{2}{r_{\mathrm{x}}^{2}} \int_{0}^{r_{\mathrm{x}}} r d r \cdot R(r, l, t)\right\}
$$


If we suppose that $v_{\mathrm{r}}$ is independent of $l$ in the xylem, that is, $f_{\mathrm{E}}(l)=1$ (Eqn 19) then the integration of Eqn 20 over $l$ (and divided by $\left.l_{\max }\right)$ gives at steady state $(\partial \overline{\mathrm{R}} / \partial t=0)$ :

$$
\frac{1}{r} \frac{d r \bar{v}_{\mathrm{r}} \bar{R}}{d r}+\frac{1}{l_{\max }}\left[v_{\mathrm{x}} R\right]_{0}^{l_{\max }}=D\left\{\frac{\kappa_{\mathrm{x}}}{r} \frac{d}{d r}\left(r \frac{d \bar{R}}{d r}\right)+\frac{1}{l_{\max }}\left[\frac{\partial R}{\partial l}\right]_{0}^{l_{\max }}\right\},
$$

where $\bar{R}$ denotes the mean value of $R(r, l, t)$ over the interval $\left[0, l_{\max }\right]$. In $\Delta$-notations, and using boundary conditions Eqns 21a, 21b, this reduces to

$$
\frac{d}{d r}\left\{r\left[\bar{v}_{\mathrm{r}} \bar{\Delta}-D_{\mathrm{x}} \frac{d \bar{\Delta}}{d r}\right]\right\}=0
$$

In other words, the expression between curly brackets is constant between $r=0$ and $r=r_{\mathrm{x}}^{-}$. Because this expression is proportional to $r$, if we set $r=0$, we can see that the constant must be zero:

$$
\frac{2 \wp_{\mathrm{x}}}{r_{\mathrm{x}}^{2}} r \bar{\Delta}-\frac{d \bar{\Delta}}{d r}=0
$$

with $\wp_{\mathrm{x}} \equiv \bar{v}\left(r_{\mathrm{x}}^{-}\right) r_{\mathrm{x}} / 2 \kappa_{\mathrm{x}} D$. The solution of this ordinary differential equation is

$$
\bar{\Delta}(r)=\bar{\Delta}\left(r_{\mathrm{x}}^{-}\right) \cdot e^{-\wp_{\mathrm{x}}\left[1-\left(r / r_{\mathrm{x}}\right)^{2}\right]} \text {. }
$$

Furthermore, by definition, we have $\bar{\Delta}_{\mathrm{x}} \equiv \frac{2}{r_{\mathrm{x}}^{2}} \int_{0}^{r_{\mathrm{x}}} r \bar{\Delta}(r) d r$ and the integration of Eqn A11 over $r$ gives

$\wp_{\mathrm{x}} \bar{\Delta}_{\mathrm{x}}-[\bar{\Delta}]_{0}^{r-\bar{x}}=0$.

The combination of the last two equations leads to

$$
\bar{\Delta}_{\mathrm{x}}=\bar{\Delta}\left(r_{\mathrm{x}}^{-}\right) \cdot \frac{1-e^{-\wp_{\mathrm{x}}}}{\wp_{\mathrm{x}}}=\bar{\Delta}\left(r_{\mathrm{x}}^{-}\right) \cdot f_{\mathrm{xx}}
$$

\section{Bulk isotope ratio in the mesophyll}

The bulk isotope ratio in the mesophyll is computed as follows:

$$
\bar{R}_{\mathrm{m}}(t)=\frac{1}{l_{\max }} \int_{0}^{l_{\max }} d l \cdot\left\{\frac{1}{r_{\mathrm{m}}} \int_{r_{\mathrm{x}}}^{r_{\max }} d r \cdot R(r, l, t)\right\}
$$

When $v_{\mathrm{r}}$ in the mesophyll is independent of $l, f_{\mathrm{E}}(l)=1$, the integration of Eqn 22 between zero and $l_{\max }$ is straightforward and, at steady state, this leads to

$$
\frac{d}{d r}\left[\frac{\wp_{\mathrm{m}}}{r_{\mathrm{m}}} \bar{R}-\frac{d \bar{R}}{d r}\right]=0
$$

with $\wp_{\mathrm{m}} \equiv v_{\mathrm{m}} r_{\mathrm{m}} / \kappa_{\mathrm{m}} D$. In other words, the expression between brackets is constant between $r=r_{\mathrm{x}}$ and $r=r_{\mathrm{e}}$. Using Eqn 23 , this constant is equal to $\wp_{\mathrm{m}} \bar{R}_{\mathrm{E}} / r_{\mathrm{m}}=\wp_{\mathrm{m}} R_{\mathrm{s}} / r_{\mathrm{m}}$, that is, zero in $\Delta$-notation: $\frac{\wp_{\mathrm{m}}}{r_{\mathrm{m}}} \bar{\Delta}-\frac{d \bar{\Delta}}{d r}=0$

The solution of this ordinary differential equation is

$$
\bar{\Delta}(r)=\Delta_{\mathrm{C}} \cdot e^{-\wp_{\mathrm{m}}\left[1-\frac{r-r_{\mathrm{x}}}{r_{\max }}\right]} .
$$

Integrated between $r=r_{\mathrm{x}}$ and $r=r_{\mathrm{e}}$, this gives

$$
\bar{\Delta}_{\mathrm{m}}=\Delta_{\mathrm{C}} \cdot \frac{1-e^{-\wp_{\mathrm{m}}}}{\wp_{\mathrm{m}}} .
$$

\section{Bulk isotope ratio}

Now if we define the bulk isotope ratio as

$$
\bar{R}_{\mathrm{lw}}=\frac{a_{\mathrm{x}} l_{\max } \cdot \bar{R}_{\mathrm{x}}+2 r_{\mathrm{m}} s_{\mathrm{x}} l_{\mathrm{max}} \cdot \bar{R}_{\mathrm{m}}}{a_{\mathrm{x}} l_{\max }+2 r_{\mathrm{m}} s_{\mathrm{x}} l_{\max }}=\phi_{\mathrm{x}} \cdot \bar{R}_{\mathrm{x}}+\left(1-\phi_{\mathrm{x}}\right) \cdot \bar{R}_{\mathrm{m}},
$$

then, in $\Delta$-notation this leads to

$$
\begin{aligned}
\bar{\Delta}_{\mathrm{lw}}= & \phi_{\mathrm{x}} \cdot \bar{\Delta}_{\mathrm{x}}+\left(1-\phi_{\mathrm{x}}\right) \cdot \bar{\Delta}_{\mathrm{m}}=\left[\phi_{\mathrm{x}} \cdot f_{\mathrm{xx}} \frac{\bar{\Delta}\left(r_{\mathrm{x}}^{-}\right)}{\Delta_{\mathrm{C}}}+\right. \\
& \left.\left(1-\phi_{\mathrm{x}}\right) \cdot f_{\mathrm{em}}\right] \cdot \Delta_{\mathrm{C}} .
\end{aligned}
$$

Now if we assume that the isotope ratio is continuous at $r=r_{\mathrm{x}}$, (which is at least approximately true) then from Eqn A18 we have $\bar{\Delta}\left(r_{\mathrm{x}}^{-}\right)=\bar{\Delta}\left(r_{\mathrm{x}}^{+}\right)=\Delta_{\mathrm{C}} \cdot e^{-\wp_{\mathrm{m}}}$ and this leads exactly to Eqn 27 in the main text.

\section{Bulk non-steady-state isotope ratios assuming $f_{\mathrm{E}}(I)=1$ (Eqn 30)}

\section{Bulk isotope ratio in the xylem}

We suppose again that $v_{\mathrm{r}}$ is independent of $l$ in the xylem, that is, $f_{\mathrm{E}}(l)=1$. Then the integration of Eqn 20 over $l$ gives

$$
\begin{aligned}
& \frac{\partial \bar{R}}{\partial t}+\frac{1}{l_{\max }}\left[v_{\mathrm{x}} R\right]_{0}^{l_{\max }}+\frac{1}{r} \frac{\partial r \bar{v}_{\mathrm{r}} \bar{R}}{\partial r}= \\
& D\left\{\frac{1}{l_{\max }}\left[\frac{\partial R}{\partial l}\right]_{0}^{l_{\max }}+\frac{\kappa_{\mathrm{x}}}{r} \frac{\partial}{\partial r}\left(r \frac{\partial \bar{R}}{\partial r}\right)\right\}
\end{aligned}
$$

with

$\bar{v}_{\mathrm{r}}(r, t)=\frac{s_{\mathrm{x}}}{2 \pi r_{\mathrm{x}}} \cdot \frac{\bar{E}(t)}{C} \cdot \frac{r}{r_{\mathrm{x}}}$.

Using boundary conditions Eqns 21a and 21b and noting that $v_{\mathrm{x}}$ is zero at $l=l_{\max }$ leads to

$$
\frac{\partial \bar{R}}{\partial t}=\frac{1}{l_{\max }} v_{\mathrm{x} 0} R_{\mathrm{s}}+\frac{1}{r}\left\{D_{\mathrm{x}} \frac{\partial}{\partial r}\left(r \frac{\partial \bar{R}}{\partial r}\right)-\frac{\partial r \bar{v}_{\mathrm{r}} \bar{R}}{\partial r}\right\} .
$$

Then, multiplying by $2 \pi r l_{\max }$ and performing a second integration over $r$ gives 


$$
\begin{aligned}
a_{\mathrm{x}} l_{\max } \cdot \frac{d \bar{R}_{\mathrm{x}}}{d t}= & a_{\mathrm{x}} \cdot v_{\mathrm{x} 0} R_{\mathrm{s}}-2 \pi r_{\mathrm{x}} l_{\max } \cdot \\
& \left\{\bar{v}_{\mathrm{r}}\left(r_{\mathrm{x}}^{-}, t\right) \bar{R}\left(r_{\mathrm{x}}^{-}, t\right)-\left.D_{\mathrm{x}} \frac{\partial \bar{R}}{\partial r}\right|_{\overline{\mathrm{x}}_{\overline{\mathrm{x}}}, t}\right\} .
\end{aligned}
$$

\section{Bulk isotope ratio in the mesophyll}

When $v_{\mathrm{r}}$ in the mesophyll is independent of $l, f_{\mathrm{E}}(l)=1$, the integration of Eqn 22 between zero and $l_{\max }$ is straightforward. Then, a second integration of this equation between $r=r_{\mathrm{x}}$ and $r=r_{\mathrm{e}}$ gives

$\int_{r_{\mathrm{x}}}^{r_{\mathrm{e}}} d r \frac{\partial \bar{R}}{\partial t}+\left[\bar{v}_{\mathrm{r}} \cdot \bar{R}\right]_{r_{\mathrm{x}}^{+}}^{r_{\mathrm{e}}}=D_{\mathrm{m}}\left[\frac{\partial \bar{R}}{\partial r}\right]_{r_{\mathrm{x}}^{+}}^{r_{\mathrm{e}}}$.

Multiplying the latter equation by $2 s_{x} l_{\max }$ and using Eqn 23 gives

$$
\begin{aligned}
2 r_{\mathrm{m}} s_{\mathrm{x}} l_{\max } \cdot \frac{d \bar{R}_{\mathrm{m}}}{d t}= & 2 s_{\mathrm{x}} l_{\max } \cdot\left\{\bar{v}_{\mathrm{r}}\left(r_{\mathrm{x}}^{+}, t\right) \bar{R}\left(r_{\mathrm{x}}^{+}, t\right)-\right. \\
& \left.\left.D_{\mathrm{m}} \frac{\partial \bar{R}}{\partial r}\right|_{r_{\mathrm{x}}^{+}, t}\right\}-2 s_{\mathrm{x}} l_{\max } \cdot \bar{v}_{\mathrm{r}}\left(r_{\mathrm{e}}, t\right) \bar{R}_{\mathrm{E}} .
\end{aligned}
$$

\section{Bulk isotope ratio}

Combining Eqn A25 and Eqn A27 and using Eqn 25, we obtain $\frac{d \bar{R}_{\mathrm{lw}}}{d t}=\frac{\phi_{\mathrm{x}}}{l_{\max }} \cdot v_{\mathrm{x} 0} R_{\mathrm{s}}-\frac{1-\phi_{\mathrm{x}}}{r_{\mathrm{m}}} \cdot \bar{v}_{\mathrm{r}}\left(r_{\mathrm{e}}, t\right) \bar{R}_{\mathrm{E}}(t)$

or, with $W=a_{\mathrm{x}} l_{\max }+2 r_{\mathrm{m}} S_{\mathrm{x}} l_{\max }$ :

$W \frac{d \bar{R}_{\mathrm{lw}}}{d t}=a_{\mathrm{x}} \cdot v_{\mathrm{x} 0} R_{\mathrm{s}}-2 s_{\mathrm{x}} l_{\max } \cdot \bar{v}_{\mathrm{r}}\left(r_{\mathrm{e}}, t\right) \bar{R}_{\mathrm{E}}(t)$

In $\Delta$-notation, this leads to

$W \frac{d \bar{\Delta}_{\mathrm{lw}}}{d t}=-2 s_{\mathrm{x}} l_{\max } \cdot \bar{v}_{\mathrm{r}}\left(r_{\mathrm{e}}, t\right) \bar{\Delta}_{\mathrm{E}}(t)$.

Equation 4, averaged over the leaf surface, becomes

$\bar{R}_{\mathrm{E}}=\frac{\bar{R}_{\mathrm{e}}-h \alpha^{+} R_{\mathrm{v}}}{\alpha_{\mathrm{k}} \alpha^{+}(1-h)}$.

In $\Delta$-notation and using Eqn 5 this gives

$\bar{\Delta}_{\mathrm{E}}=\frac{\bar{\Delta}_{\mathrm{e}}-\Delta_{\mathrm{C}}}{\alpha_{\mathrm{k}} \alpha^{+}(1-h)}$.

Combined with Eqn A30, this leads exactly to Eqn 30 in the main text if $W_{\mathrm{lw}}\left(\mathrm{mol} \mathrm{m}^{-2}\right)$ is equal to $C W /\left(2 s_{\mathrm{x}} l_{\max }\right)$. 